\title{
FORMULAE AND ASYMPTOTICS FOR COEFFICIENTS OF ALGEBRAIC FUNCTIONS
}

\author{
CYRIL BANDERIER AND MICHAEL DRMOTA
}

\begin{abstract}
** We dedicate this article to the memory of Philippe Flajolet, who was and will remain a guide and a wonderful source of inspiration for so many of us. ****
\end{abstract}

[ This article will appear in Combinatorics, Probability, and Computing, in the special volume dedicated to Philippe Flajolet. ]

Cyril Banderier, CNRS/Univ. Paris 13, Villetaneuse (France).

Cyril.Banderier at lipn.univ-paris13.fr, http://lipn.univ-paris13.fr/ banderier

Michael Drmota, TU Wien (Austria).

drmota at dmg.tuwien.ac.at, http://dmg.tuwien.ac.at/drmota/

Date: March 22, 2013 (revised March 22, 2014).

Key words and phrases. analytic combinatorics, generating function, algebraic function, singularity analysis, context-free grammars, critical exponent, non-strongly connected positive systems, Gaussian limit laws, $\mathbb{N}$-algebraic function. 
Abstract. We study the coefficients of algebraic functions $\sum_{n \geq 0} f_{n} z^{n}$. First, we recall the too-little-known fact that these coefficients $f_{n}$ always admit a closed form. Then we study their asymptotics, known to be of the type $f_{n} \sim C A^{n} n^{\alpha}$. When the function is a power series associated to a contextfree grammar, we solve a folklore conjecture: the critical exponents $\alpha$ cannot be $1 / 3$ or $-5 / 2$; they in fact belong to a proper subset of the dyadic numbers. We initiate the study of the set of possible values for $A$. We extend what Philippe Flajolet called the Drmota-Lalley-Woods theorem (which states that $\alpha=-3 / 2$ when the dependency graph associated to the algebraic system defining the function is strongly connected). We fully characterize the possible singular behaviors in the non-strongly connected case. As a corollary, the generating functions of certain lattice paths and planar maps are not determined by a context-free grammar (i.e., their generating functions are not $\mathbb{N}$-algebraic). We give examples of Gaussian limit laws (beyond the case of the Drmota-Lalley-Woods theorem), and examples of non-Gaussian limit laws. We then extend our work to systems involving non-polynomial entire functions (non-strongly connected systems, fixed points of entire functions with positive coefficients). We give several closure properties for $\mathbb{N}$-algebraic functions. We end by discussing a few extensions of our results (infinite systems of equations, algorithmic aspects).

RÉSumÉ. Cet article a pour héros les coefficients des fonctions algébriques. Après avoir rappelé le fait trop peu connu que ces coefficients $f_{n}$ admettent toujours une forme close, nous étudions leur asymptotique $f_{n} \sim C A^{n} n^{\alpha}$. Lorsque la fonction algébrique est la série génératrice d'une grammaire noncontextuelle, nous résolvons une vieille conjecture du folklore : les exposants critiques $\alpha$ ne peuvent pas être $1 / 3$ ou $-5 / 2$ et sont en fait restreints à un sousensemble des nombres dyadiques. Nous amorçons aussi l'étude de l'ensemble des valeurs possibles pour $A$. Nous étendons ce que Philippe Flajolet appelait le théorème de Drmota-Lalley-Woods (qui affirme que $\alpha=-3 / 2$ dès lors qu'un "graphe de dépendance" associé au système algébrique est fortement connexe) : nous caractérisons complètement les exposants critiques dans le cas non fortement connexe. Un corolaire immédiat est que certaines marches et cartes planaires ne peuvent pas être engendrées par une grammaire noncontextuelle non ambigüe ( $i$. e., leur série génératrice n'est pas $\mathbb{N}$-algébrique). Nous donnons un critère pour l'obtention d'une loi limite gaussienne (cas non couvert par le théorème de Drmota-Lalley-Woods), et des exemples de lois non gaussiennes. Nous étendons nos résultats aux systèmes d'équations de degré infini (systèmes non fortement connexes impliquant des points fixes de fonctions entières à coefficients positifs). Nous donnons quelques propriétés de clôture pour les fonctions $\mathbb{N}$-algébriques. Nous terminons par diverses extensions de nos résultats (systèmes infinis d'équations, aspects algorithmiques). 


\section{Contents}

1. Introduction 3

2. Definitions: $\mathbb{N}$-algebraic functions and well defined systems 6

3. Closed form for the coefficients of algebraic functions 12

4. Critical exponents for coefficients of algebraic function $\quad 15$

5. Finer asymptotics for $\mathbb{R}_{+}$-algebraic functions 18

5.1. Well defined versus well posed systems of functional equations $\quad 18$

5.2. Critical exponents of $\mathbb{R}_{+}$-algebraic functions $\quad 19$

5.3. Dependency graph and auxiliary results 20

5.4. Proof of our Theorem 4 on the possible Puiseux expansions 23

6. Periodicities 27

7. Possible radius of convergence of $\mathbb{Q}_{+}$-algebraic functions 34

8. Limit laws 37

8.1. The classical Drmota-Lalley-Woods theorem 37

8.2. More Gaussian examples, beyond the Drmota-Lalley-Woods case 37

8.3. Non-Gaussian limit laws 38

9. Beyond the algebraic case: positive systems of entire functions 39

10. Conclusions 41

$\begin{array}{ll}\text { References } & 42\end{array}$

\section{INTRODUCTION}

Algebraic functions and their asymptotics are ubiquitous in combinatorics; we review here some of the main reasons for this.

Algebraic functions and language theory. The theory of context-free grammars and its relationship with combinatorics was initiated by the article of Noam Chomsky and Marcel-Paul Schützenberger in 1963 [38], where it is shown that the generating function of the number of words generated by a non-ambiguous contextfree grammar is algebraic. Since then, there has been much use of context-free grammars in combinatorics; several chapters of the Flajolet \& Sedgewick book "Analytic Combinatorics" [63] are dedicated to what they called the "symbolic method" (which is in large parts isomorphic to the Joyal theory of species [71, 20], and when restricted to context-free grammars, it is sometimes called the "DVS methodology", for Delest-Viennot-Schützenberger, as the Bordeaux combinatorics school indeed made a deep use of it $[114,46]$, e.g. for enumeration of polyominoes [47] and lattice paths [58]). Context-free grammars also allow one to enumerate trees [66, 87], sofic-Dyck automata [18], non-commutative identities [78, 95], pattern-avoiding permutations [77, 85, 3, 17], some type of planar maps, triangulations, Apollonian networks [26], non-crossing configurations, dissections of polygons [61] (as studied by Euler in 1751, one of the founding problems of analytic combinatorics!), see also [107]. Links between asymptotics of algebraic functions and the (inherent) ambiguity of context-free languages was studied in [74,60], and for prefixes of infinite words in [5]. Growth rates are studied in [35, 36], in connection with asymptotics of random walks $[117,37,67,81,82]$. Applications in bio-informatics or for patterns in RNA are given in [93, 115, 49, 41, 116]. 
Random generation of algebraic objects. The first efficient algorithm to make uniform random generation of words of length $n$ of a context-free grammar involved $n^{2}(\ln n)^{2}$ operations on average via fast Fourier transform and is due to Hickey and Cohen [70]. Generating functions associated to context-free grammars are the key tool to improve this average complexity: the recursive method, coupled with the boustrophedon algorithm [64], lead to a time-complexity $n \ln n$. Asymptotics of their coefficients are used in the implementation of this method with floating-point arithmetic [50]. The case of ambiguous grammars was also considered in [24]. A revolutionary change of paradigm on the generating functions was done in [59], where the authors introduced what they called the "Boltzmann method"; this lead to $O(n)$ complexity to generate objects of size $n \pm \epsilon(n)$, benefitting new analytic investigations on the function. See also applications of this method to algebraic objects in [45, 34] or grammars with parameters in [27].

Other occurrences of algebraic functions. The link between algebraic functions and p-automatic sequences (numbers written in a given base via an automata) is well illustrated by the Christol-Kamae-Mendès France-Rauzy theorem [39, 4].

More links with monadic second-order logic, tiling problems and vector addition systems appears in $[90,118]$.

Algebraic functions via functional equations. Many algebraic functions pop up in combinatorics [31] via tools other than context-free grammars: quite often, they appear as the "diagonal" of rational functions $[13,15]$, or as solutions of functional equations (solvable by the "kernel method" and its variants $[9,32]$ ), and the interplay with their asymptotics is crucial for analysis of lattice paths [10], walks with an infinite set of jumps [7, 14] (which are thus not coded by a grammar on a finite alphabet), or planar maps [11]. Sometimes, differential equations lead to algebraic solutions, like in some urn models [88].

Algebraicity of permutation related problems. Since Knuth enumerated permutations sortable by a stack, much attention has been drawn to permutations avoiding a given pattern, or counting the number of appearances of a given pattern. Many cases involved a Knuth-like approach (using the "kernel method"), or an approach by (in)decomposable subclasses [77, 85, 3, 17], leading to algebraic functions.

Algebraic functions and universality of critical exponents. When one thinks about the asymptotics of the coefficients of generating functions, one often gives the example of Catalan numbers $\left(\begin{array}{c}2 n \\ n\end{array}\right) /(n+1) \sim 4^{n} / \sqrt{\pi n^{3}}$ (this is a direct consequence of Stirling's formula for $n$ !, or can also be obtained by the saddle point method or singularity analysis). One important fact with respect to the asymptotics of coefficients of algebraic generating functions, is that it often involves the factor $1 / \sqrt{\pi n^{3}}$. This was called in [63] the "Drmota-Lalley-Woods theorem", due to independent similar results of these three authors [52, 81, 118], relying on a "strong connectivity" assumption of the system implicitly defining the algebraic function. In this article, we extend this theorem by removing this restrictive assumption, to give all the possible asymptotics for the important class of context-free algebraic functions, and associated limit laws. In one sense, this could be considered as the generalization of Perron-Frobenius theorem to the algebraic case, including also a multivariate extension. We do this in a constructive way, which opens the door to full algorithmization. 


\section{Plan of this article:}

- In Section 2, we give a few definitions, mostly illustrating the link between context-free grammars, solutions of positive algebraic systems, and $\mathbb{N}$-algebraic functions. We also prove some basic properties of such functions.

- In Section 3, we survey some closure properties of algebraic functions and give a closed form for their coefficients.

- In Section 4, we state our main theorem on the possible critical exponents of algebraic functions (associated to a context-free grammar with positive weights) and we give some consequences of it.

- In Section 5, we give finer results about asymptotics and the corresponding Puiseux expansions, which incidentally prove our main theorem.

- In Section 6, we provide a complete picture for the asymptotic behavior of the coefficients of functions that are solutions of positive systems of algebraic equations when a "periodic behavior" occurs.

- In Section 7, we consider the mysterious set of all the radii of convergence of $\mathbb{N}$-algebraic functions, and some variants. We give some closure properties of this set.

- In Section 8, we prove that the associated limit laws are Gaussian for a broad variety of cases (thus extending the Drmota-Lalley-Woods theorem), and we also give an argument explaining the diversity of other possible limit laws.

- In Section 9, we give an analog of our main theorem for systems involving entire functions.

- We end with a conclusion pinpointing some extensions (algorithmic considerations, decidability questions, extension to infinite systems, to attribute grammars).
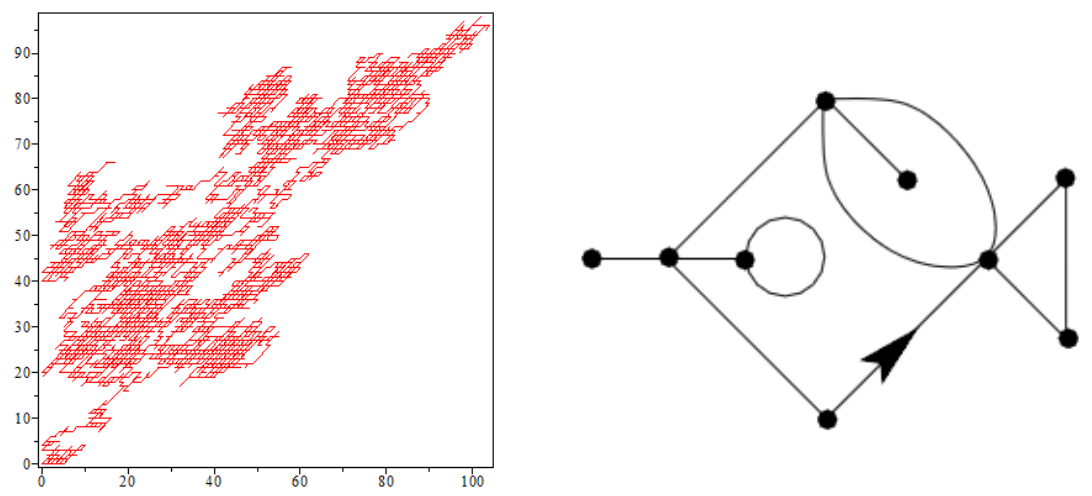

Figure 1. Many combinatorial objects are algebraic. Sometimes, it is for some non-trivial reasons, like e.g. Gessel random walks (left) or planar maps (right). A nice consequence of our Theorem 3 is that these combinatorial structures (and many similar objects) cannot be generated by a context-free grammar, because of their asymptotics have the critical exponent $-2 / 3$ and $-5 / 2$. 


\section{Definitions: $\mathbb{N}$-ALGEBRAiC FUnCtions AND WELl DEFined SyStems}

Coefficients of algebraic functions have a very constrained asymptotics; we will focus on their "critical exponents":

Definition 1 (Critical exponent of a sequence). For any sequence such that $f_{n} \sim$ $C n^{\alpha} \rho^{-n}, \alpha$ is called the critical exponent and $\rho$ is the radius of convergence of the corresponding generating function $F(z)=\sum_{n \geq 0} f_{n} z^{n}$. Moreover, if the sequence $f_{n}$ has asymptotics depending on the modulo class of $n$, the definition extends naturally by saying that $f_{n}$ has a (possibly different) critical exponent for each class. The singularities of $F$ located on its radius of convergence are called the dominant singularities of $F$.

There is a dual point of view when one considers functions instead of sequences:

Definition 2 (Puiseux critical exponent of a function). For any function having a Puiseux expansion $F(z)=\sum_{k \geq k_{0}} a_{k}(z-\rho)^{r k}$ (with $a_{k_{0}} \neq 0$ ), the number $r k_{0}$ is called the valuation of this series, while the smallest value of rk which is not a nonnegative integer and for which $a_{k} \neq 0$ is called the Puiseux critical exponent of $F(z)$. In this article, $\rho$ is by default assumed to be the radius of convergence of $F(z)$.

Examples: $F(z)=1+2(1-z)+5(1-z)^{2}+(1-z)^{5 / 2}+(1-z)^{3}+(1-z)^{7 / 2}(1+o(1))$ has Puiseux critical exponent 5/2. $F(z)=1 /(1-2 z)+(1-2 z)+(1-2 z)^{5 / 2}(1+o(1))$ has Puiseux critical exponent -1 .

The Flajolet-Odlyzko theory of singularity analysis [62] (which has roots in the works of Darboux, Hankel, Hardy and Littlewood, ... ) links the critical exponent $\alpha$ of a sequence $\left(f_{n}\right)$ and the Puiseux critical exponent $e$ of the corresponding function $F(z)$ via the relation $\alpha=-e-1$ (provided that $\rho$ is the only singularity on the circle $|z|=\rho$ ).

We will consider algebraic functions defined via systems of algebraic equations. Such systems are ubiquitous in language theory. For the notions of automata, pushdown automata, context-free grammars, we refer to the first three chapters of [113] (by Perrin on finite automata, by Berstel and Boasson on context-free languages, by Salomaa [98] on formal languages and power series) or to the more recent survey [92] in [56]. Another excellent compendium on the subject is the handbook of formal languages [97] and the Lothaire trilogy [84]. In language theory, it is natural to consider equations involving positive integer coefficients, whereas for other applications it is natural to consider positive real weights or probabilities. Accordingly, we will consider equations having various types of coefficients:

Definition 3 (Well-posed systems and $\mathbb{N}$-algebraic functions, $\mathbb{Q}_{+}$-algebraic functions, ...). A "well posed" system is a system ${ }^{1}$

$$
\left\{\begin{array}{l}
y_{1}=P_{1}\left(z, y_{1}, \ldots, y_{d}\right) \\
\vdots \\
y_{d}=P_{d}\left(z, y_{1}, \ldots, y_{d}\right)
\end{array}\right.
$$

where each polynomial $P_{i}$ is such that $\left[y_{i}\right] P_{i} \neq 1$ and has coefficients in any set $\mathbb{S}$ of real numbers that is closed under addition and multiplication (in this article, we

\footnotetext{
${ }^{1}$ In this article, we will often abbreviate the system (1) with the convenient short notation $\mathbf{y}=\mathbf{P}(z, \mathbf{y})$, where bold fonts are used for vectors.
} 
consider $\mathbb{S}=\mathbb{N}, \mathbb{Z}, \mathbb{Q}_{+}$, or $\left.\mathbb{R}_{+}\right)^{2}$ and which has a tuple of solution $\left(f_{1}(z), \ldots, f_{d}(z)\right)$, where each $f_{i}(z)$ is a power series with coefficients in $\mathbb{S}$. Functions solutions of such a system are called $\mathbb{S}$-algebraic functions.

$$
\begin{aligned}
& \left\{\begin{array} { l } 
{ y _ { 1 } = 3 y _ { 2 } y _ { 1 } + 4 z } \\
{ y _ { 2 } = 2 3 y _ { 1 } ^ { 2 } + 5 z }
\end{array} \quad \left\{\begin{array}{l}
y_{1}=\frac{1}{3} z y_{2}+\frac{25}{4} z y_{1}+z \\
y_{2}=z y_{1}+z y_{2}
\end{array}\right.\right. \\
& \left\{\begin{array} { l } 
{ y _ { 1 } = 3 y _ { 2 } y _ { 1 } + 4 z } \\
{ y _ { 2 } = 2 3 y _ { 1 } - 5 z }
\end{array} \quad \left\{\begin{array}{l}
y_{1}=3.14 z y_{1} y_{2}+\pi z^{3} y_{1} y_{3} \\
y_{2}=\exp (-1) y_{1} y_{2}+z^{3} \\
y_{3}=z+\cos (3) y_{3}^{2}
\end{array}\right.\right.
\end{aligned}
$$

EXAMPLES 1. The solutions of the above systems are examples of functions which are $\mathbb{N}$-algebraic (left, top), $\mathbb{Z}$-algebraic (left, bottom), $\mathbb{Q}_{+}$-algebraic (right, top), and $\mathbb{R}_{+}$-algebraic (right, bottom).

Remark: requiring that the solutions have real coefficients is indeed a restriction, as e.g. the equation $y=1+z+y^{2}$ has two power series solutions, but each of them has complex coefficients. On the other hand, if the system is with positive coefficients and such that $\left[y_{i}\right] P_{i}=0$ and $P_{i}(0, \mathbf{0})=0$ for all $i$, then it would be superfluous to require that the $y_{i}$ 's have nonnegative real coefficients, as this would result by iteration (while a system like $y=z+2 y$ shows that the power series could elsewhere have negative coefficients as soon as there is one $i$ such that $\left[y_{i}\right] P_{i}>1$ ). Similarly, when one considers just $\mathbb{N}$-algebraic functions, one could also change the condition $\left[y_{i}\right] P_{i} \neq 1$ by the (apparently more restrictive) condition $\left[y_{i}\right] P_{i}=0$; this would not actually change the set of $\mathbb{N}$-algebraic functions (this easily follows from our Proposition 2 hereafter).

We now give a definition which offers a fast "combinatorial" way to ensure that a system is well posed:

Definition 4 (Well defined systems). The above system (1), $\mathbf{y}=\mathbf{P}(z, \mathbf{y})$, is called "well defined" if and only if

- (no monic production) the coefficient of each $y_{j}$ in each $P_{i}$ is 0 : $\forall i \forall j \quad\left[y_{j}\right] P_{i}\left(0,0, \ldots, 0, y_{j}, 0, \ldots, 0\right)=0$,

- (no epsilon production) for all $i, P_{i}(0,0, \ldots, 0)=0$,

- (terminating condition) for any $y_{j}$, there is at least one $y_{k}$ reachable from $y_{j}$ (i.e., $\exists t>0 \mid \mathbf{A}_{j k}^{t} \neq 0$, where $\mathbf{A}$ is the adjacency matrix of the dependency graph associated to the system, as illustrated by Figure 2 in Section 5.4), such that $P_{k}(z, 0, \ldots, 0) \neq 0$.

By design, any well defined system is well posed, but the converse is not true, as can be seen from systems of Examples 2. We will discuss some analytic distinctions between these two notions in Section 5.1, as introducing both of them allow us to simplify the wordings of our theorems, without losing generality on the combinatorial or analytical problems we want to consider.

Remark. Several authors introduced different notions with some minor distinctions: our "well defined" systems are thus related to the "proper" or "well posed" or "well founded" notions of $[38,52,94]$.

\footnotetext{
${ }^{2}$ We make use of the notations $\mathbb{Q}_{+}=\{x \in \mathbb{Q}, x \geq 0\}, \mathbb{R}_{+}=\{x \in \mathbb{R}, x \geq 0\}$, and $\left[u^{k}\right] P(u)$, which stands for the coefficient of $u^{k}$ in $P(u)$.
} 


$$
\begin{array}{ll} 
\begin{cases}y_{1}=\frac{1}{2} y_{1}+\frac{1}{2} y_{2}+z \\
y_{2}=\frac{1}{2} y_{1}+\frac{1}{2} y_{2}+z\end{cases} & \left\{\begin{array}{l}
y=z+2 y \\
y_{2}=1+z+z y_{2}+y_{3} \\
y_{3}=5 y_{4} \\
y_{4}=\frac{1}{10} y_{3} y_{4}
\end{array}\right. \\
\left\{\begin{array}{l}
y=1+z+y^{2} \\
y_{2}=2 y_{3} \\
y_{3}=\frac{1}{2} y_{2}+y_{3}^{2}+z
\end{array}\right. & \left\{\begin{array}{l}
y_{1}=1+y_{1} y_{3}^{4} \\
y_{2}=3 y_{2}+z y_{1}+z \\
y_{3}=z y_{2}^{2}+z y_{1}+z
\end{array}\right.
\end{array}
$$

EXAMPLES 2. The above list shows systems that are not well defined. Well defined systems (our Definition 4) are just a convenient way to prevent pathological cases like the examples on the two first columns. The systems from the right column are not well defined, but are "well posed" (our Definition 3): they have power series solutions with nonnegative real coefficients.

The idea is always that it corresponds to context-free grammars for which one has no "infinite chain rules" (no infinite chain of monic productions, no infinite chain of epsilon productions).

In this article, we need this to handle probability generating functions (in order to get general results on the limit laws), therefore we allow real weights. So the situation is slightly more tricky than what previous authors considered, as they were just dealing with systems having integer coefficients; indeed, in the case of real coefficients, "analytic convergence" can compensate a "formal divergence" (as for example for the grammar $A \rightarrow z A / 2$ ); this is why (in the notion of well posed system), one allows the coefficients of monic productions to be $\neq 0$, as soon as the spectral radius of the Jacobian of the associated system is $<1$, as this will guaranty a global contraction (we will also comment on this when discussing our notion of well posed systems in our subsection 5.1; see also [111] for a more topological point of view). It is true that the two rules "no monic productions" and "no epsilon productions" are a little bit more restrictive than what is needed (as illustrated by the first case in the right column of our Examples 2), but they make it easy to test the "well definedness" of a system; what is more, for the systems not fulfilling all the conditions of Definition 4 which nonetheless have an analytic and combinatorial meaning, it is easy to transform them into a system fulfilling these conditions (essentially by replacing some coefficients of the polynomials $P_{i}$ by some new formal parameters).

A little caveat. We mentioned the matter of formal convergence; let us recall briefly that this is defined on the set of power series by the following ultrametric distance:

$$
d(F(z), G(z)):=2^{-\operatorname{val}(F(z)-G(z))}
$$

(val giving the exponent of the first non-zero monomial). This distance extends to vectors of functions (and to multivariate series), and allows to apply the Banach fixed-point theorem: the system (1) being a contraction implies existence and uniqueness of a solution of the system as a $d$-tuple of power series $\left(y_{1}, \ldots, y_{d}\right)$ (and they are analytic functions near 0 , as we already know that they are power series 
and algebraic by nature). A common mistake is to forget that there exist situations for which the system (1) can admit several solutions as power series for $y_{1}$ (nota bene: there is no contradiction with our previous claim, which is considering tuples). We illustrate this point via the system

$$
\left\{\begin{array}{l}
y_{1}=z\left(1+y_{2}+2 y_{1}^{2}\right) \\
y_{2}=z\left(1+y_{1}+y_{2}^{2}\right)
\end{array} .\right.
$$

If one eliminates $y_{2}$ in this system, this gives that $y_{1}$ is defined by the equation

$$
4 z^{2} y_{1}^{4}-4 z y_{1}^{3}+\left(2 z+1+4 z^{2}\right) y_{1}^{2}+\left(z^{2}-1-2 z\right) y_{1}+2 z^{2}+z=0,
$$

which has four solutions for $y_{1}: z+z^{2}+O\left(z^{3}\right), 1+3 z+10 z^{2}+O\left(z^{3}\right), \frac{1}{2} z^{-1}-$ $\frac{3}{2} z-\frac{5}{4} z^{2}+O\left(z^{3}\right)$, and $\frac{1}{2} z^{-1}-1-\frac{5}{2} z-\frac{39}{4} z^{2}+O\left(z^{3}\right)$. Here it is the case that several of these solutions are power series, and it can even be proven that two branches of Equation (3) are power series with positive integer coefficients (this is not a contradiction with unicity of the solution in the Banach fixed-point theorem, as Equation (3) is not a contraction in $\mathbb{Q}[[z]])$. The analogous elimination of $y_{1}$ in the system leads to four solutions for $y_{2}: z+z^{2}+O\left(z^{3}\right), 1+3 z+10 z^{2}+O\left(z^{3}\right)$, $\frac{1}{2} z^{-1}-\frac{3}{2} z-\frac{5}{4} z^{2}+O\left(z^{3}\right)$, and $\frac{1}{2} z^{-1}-1-\frac{5}{2} z-\frac{39}{4} z^{2}+O\left(z^{3}\right)$. However, because of the multidimensional fixed-point theorem, amongst the four pairs $\left(y_{1}, y_{2}\right)$ of functions satisfying the system, there is only one pair of power series (namely the one with $\left.y_{1} \sim y_{2} \sim z\right)$ :

$$
\begin{gathered}
\left(\begin{array}{l}
y_{1}(z) \\
y_{2}(z)
\end{array}\right)=\left(\begin{array}{c}
1+3 z+10 z^{2}+O\left(z^{3}\right) \\
\frac{1}{z}-2 z+3 z^{2}+O\left(z^{3}\right)
\end{array}\right),\left(\begin{array}{l}
y_{1}(z) \\
y_{2}(z)
\end{array}\right)=\left(\begin{array}{c}
z+z^{2}+3 z^{3}+O\left(z^{4}\right) \\
z+z^{2}+2 z^{3}+O\left(z^{4}\right)
\end{array}\right), \\
\left(\begin{array}{l}
y_{1}(z) \\
y_{2}(z)
\end{array}\right)=\left(\begin{array}{c}
\frac{1}{2 z}-1-\frac{5}{2} z+O\left(z^{2}\right) \\
\frac{1}{z}-\frac{1}{2}-\frac{1}{4} z+O\left(z^{2}\right)
\end{array}\right), \text { and }\left(\begin{array}{l}
y_{1}(z) \\
y_{2}(z)
\end{array}\right)=\left(\begin{array}{c}
\frac{1}{2 z}-\frac{3}{2} z-\frac{5}{4} z^{2}+O\left(z^{3}\right) \\
\frac{1}{2}-\frac{5}{4} z-\frac{1}{4} z^{2}+O\left(z^{3}\right)
\end{array}\right) .
\end{gathered}
$$

Different algebraic classes. By elimination theory (resultant or Gröbner bases, see the discussion on this in $[108,91])$, S-algebraic functions are algebraic functions: it is possible to transform a system of equations in the $y_{i}$ 's in a single equation involving just $y_{1}$ and $z$. Now we give a few trivial/folklore results: $\mathbb{N}$ algebraic functions correspond to generating functions of context-free grammars (this is the Chomsky-Schützenberger theorem, see [38]), or, equivalently, pushdown automata (via e.g. a Greibach normal form). They are closed by sum, product, derivation. $\mathbb{Z}$-algebraic functions have no natural simple combinatorial structures associated to them, but they are related to $\mathbb{N}$-algebraic functions via the following proposition:

Proposition 1. Any $\mathbb{Z}$-algebraic function is the difference of two $\mathbb{N}$-algebraic functions.

Proof. This can be seen by introducing two new sets of unknowns $\mathbf{a}$ and $\mathbf{b}$, and then by splitting in two the initial system:

$$
\mathbf{y}=P(z, \mathbf{y}) \Longleftrightarrow \mathbf{a}-\mathbf{b}=P(z, \mathbf{a}-\mathbf{b}) .
$$

Indeed, expanding and collecting the positive terms gives an $\mathbb{N}$-algebraic equation for $\mathbf{a}$, and collecting the negative terms gives an $\mathbb{N}$-algebraic equation for $\mathbf{b}$ : there is clearly no monic productions in right-hand side inherited from $P(z, \mathbf{y})$. 
For example, the $\mathbb{Z}$-algebraic function defined by $F=1+3 z F^{2}-3 z^{2} F^{3}$ can be written as the difference of two $\mathbb{N}$-algebraic functions: $F=A-B$ with $A=$ $1+3 z A^{2}+3 z B^{2}+9 z^{2} A^{2} B+3 z^{2} B^{3}$ and $B=6 z A B+3 z^{2} A^{3}+9 z^{2} A B^{2}$.

Proposition 2. Any algebraic power series $F(t)$ in $\mathbb{S}[[t]]$, where $\mathbb{S}=\mathbb{R}, \mathbb{Q}$, or $\mathbb{Z}$, is in fact an $\mathbb{S}$-algebraic function in disguise. More precisely, after a possible change of variable $F \rightarrow G=t^{v} F+a, G$ is $\mathbb{S}$-algebraic, and the corresponding system has just one equation.

Proof. Let us start with an algebraic function $F(t)$ satisfying a general polynomial equation, namely $Q(t, F(t))=0$ (where $Q$ is irreducible and has real coefficients). We would like to prove that we can also define it via an equation of the type of system (1), namely $F(t)=P(t, F(t))$.

To do this, we will use a little bit of Newton polygon theory: to each monomial $F(t)^{i} t^{j}$ in $Q(t, F(t))$, one associates the point $(i, j)$. The convex hull of the set of points associated to $Q(t, F(t))$ is called the Newton polygon of $Q$. Now, imagine a source of light below this polygon, which thus floodlights all the bottom segments of the polygon (and only them). Each floodlighted segments has a slope (call it $\sigma_{k}$ ), and a length on the $x$-axis (call it $\lambda_{k}$ ). The Newton polygon theory implies that one has then $\lambda_{k}$ roots of valuation $-\sigma_{k}$. This can be checked by a "plug and identify" process.

Now if $F(t)$ is an algebraic power series of valuation $v$, the change of variable $F(t) \rightarrow t^{v} F(t)$ in its characteristic polynomial $Q(t, F(t))=0$ allows us to restrict without loss of generality to power series $F(t)$ having a non-zero constant term.

Equivalently, one of the points of the Newton polygon is $(0,0)$, and another point is $(0, m)$ for some $m$ (because if not, there would be no root with integer valuation, i.e. a power series solution). If $m=1$, we got the shape of system (1). If $m>1$, then making the change of variable $y \rightarrow y+a$ leads to a new equation, in which the coefficient of $y$ is a polynomial in $a$, and any real value of $a$ not cancelling this polynomial leads to an equation satisfying the shape of system (1).

Now, what about the coefficients of this newly found $P(t, F)$ ? Well, they are by design real, and if (at least) one of the coefficients (call it $c$ ) of this equation would not be rational, then bootstrapping the equation $F=P(t, F)$ would imply that the power series $F(t)$ would have a coefficient (required to be rational) which would also be a linear relation between this irrational coefficient $c$ and other rational numbers.

The same holds with "integer" instead of "rational"; it is however more tricky there, once we got via the previous argument a polynomial $P(t, F)$ with rational coefficients, just apply the Newton polygon theory in a " $p$-adic way": first make a simplification by the lcm (call it $\ell$ ) of the denominators of the coefficients of $P(t, F)$ (call $p$ a prime factor of this lcm), and then consider the Newton polygon (taking $F$ and $p$ as coordinates) of $\ell y=\ell P(t, F)$. There, the slope of the line corresponding to our power series solution implies that its valuation in $p$ is negative, but as we know by hypothesis it is positive, it means that there were not existing such a prime $p$, and therefore they were no pure rational coefficient in $P(t, F)$.

Remark: The above proposition does not hold for $\mathbb{S}=\mathbb{N}$, indeed, we show later in Proposition 8 that $\mathbb{N}$-algebraic functions can intrinsicly require more than one equation having nonnegative coefficients to be defined, while they can always be defined with a single equation having positive and negative coefficients. A nice consequence of the algebraicity of a power series $F(z)$ with rational coefficients is 
the Eisenstein lemma, namely that there exists an integer $b$ such that $F(b z)$ has integer coefficients only: this is obvious thanks Proposition 2, as $F(z)$ is then $\mathbb{Q}$ algebraic, and thus $F(b z)$ (with a $b$ killing all the denominators of the coefficients) is then $\mathbb{Z}$-algebraic. This is a way to prove that exp or log type functions like $\sum z^{n} / n !^{k}$ and $\sum z^{n} / n^{k}$ are transcendental (see example VII.37 in [63]).

There is a natural extension of $\mathbb{N}$-algebraic functions to a multivariate setting: the multivariate function $F(\mathbf{z})$ (for $\mathbf{z}$ a tuple of variables) is called $\mathbb{S}$-algebraic if there is a system such that $\mathbf{y}=\mathbf{P}(\mathbf{z}, \mathbf{y})$, where $\mathbf{z}=\left(z_{1}, \ldots, z_{d}\right)$ and $\mathbf{y}=\left(y_{1}, \ldots, y_{m}\right)$ are tuples and where $\mathbf{P}=\left(P_{1}, \ldots, P_{m}\right)$ is a tuple of polynomials in $d+m$ variables, with coefficients in $\mathbb{S}$.

Proposition 3 (systems of $\mathbb{N}$-algebraic equations have $\mathbb{N}$-algebraic solutions). Consider a system of equations $\mathbf{y}=\mathbf{N}(\mathbf{z}, \mathbf{y})$ where $\mathbf{y}$ and $\mathbf{z}$ are tuples, and where each $N_{i}$ is a multivariate $\mathbb{S}$-algebraic function. Then the power series $y_{i}(\mathbf{z})$ solutions of this system are $\mathbb{S}$-algebraic functions.

Proof. One has a system of $d$ equations $y_{j}=N_{j}\left(\mathbf{z}, y_{1}, \ldots, y_{d}\right)$, where each $N_{j}(\mathbf{z}, \mathbf{y})$ is itself solution of a possibly large system $\mathbf{N}_{\mathbf{j}}=\mathbf{P}_{\mathbf{j}}\left(\mathbf{z}, \mathbf{y}, \mathbf{N}_{\mathbf{j}}\right.$ ) (the reader need good eyes here to distinguish bold fonts: $\mathbf{N}_{\mathbf{j}}$ is here a tuple of which $N_{j}$ is the first component). By keeping trace of all the intermediate variables, one gets a huge system and a polynomial $A_{j}$ (with coefficients in $\mathbb{S}$ ) such that $y_{j}=A_{j}\left(\mathbf{z}, \mathbf{y}, \mathbf{N}_{\mathbf{1}}, \ldots, \mathbf{N}_{\mathbf{d}}\right.$ ); thus $y_{j}$ is $\mathbb{S}$-algebraic. Alternatively, this can be seen with the context-free grammar approach, by doing a substitution: replacing some initial terminals by some non-terminals.

There is actually a nice combinatorial example of this type of equations:

Corollary 1 ( $\mathbb{N}$-algebraicity of classes of pattern-avoiding permutations). If the generating function of the so-called "simple" permutations (associated to a pattern $P)$ is $\mathbb{N}$-algebraic, then the generating function of permutations avoiding the pattern $P$ is also $\mathbb{N}$-algebraic.

Proof. Permutations avoiding some patterns satisfy the functional equation $C=$ $(1+C)(z+S(C))+C^{2}$, where $S$ is the generating functions of the so-called "simple" permutations (see $[3,17]$ ), which is known to be $\mathbb{N}$-algebraic in many cases. For such cases, our Proposition 3 therefore implies that the generating function $C$ is itself also $\mathbb{N}$-algebraic. For sure, this is also trivially the case when there is a finite number of simple permutations.

Now, let us conclude this section by mentioning $\mathbb{N}$-rational functions: an $\mathbb{N}$ rational function is a function solution of a system (1) where each polynomial $P_{i}$ has coefficients in $\mathbb{N}$ and total degree 1. Such functions correspond to generating functions of regular expressions or, equivalently, automata (a result attributed to Kleene [76]). Their coefficients satisfy a recurrence $f_{n+d}=\sum_{i=0}^{d-1} a_{i} f_{n+i}$, with $a_{i} \in \mathbb{N}$. A lot is known about $\mathbb{N}$-rational functions, and they are well characterized by their analytic and asymptotic properties [23, 112]; our article aims at reaching a similar level of knowledge about $\mathbb{N}$-algebraic functions, but before to tackling them in Section 4, we continue with facts that hold for algebraic functions in full generality. 


\section{Closed Form For the COEFFiCients of Algebraic Functions}

A first natural question is how can we compute the $n$-th coefficient $f_{n}$ of an algebraic power series? The fastest way is relying on the theory of D-finite functions. A function $F(z)$ is D-finite if it satisfies a linear differential equation with coefficients which are polynomials in $z$; equivalently, its coefficients $f_{n}$ satisfy a linear recurrence with coefficients which are polynomials in $n$. There are numerous algorithms to deal with this important class of functions, which includes a lot of special functions from physics, number theory and also combinatorics [107].

Proposition 4 (The Abel-Tannery-Cockle-Harley-Comtet Theorem). Algebraic functions are D-finite. What is more, for an algebraic function $F(z)$ solution of a polynomial equation $Q(z, F(z))=0$ (where $Q(z, y)$ has degree $d$ in $y)$, then the dimension of the space spanned by the derivatives $\partial_{z}^{k} F(z)$ is bounded by $d$.

Proof. In combinatorics, Comtet popularized the fact that algebraic functions are D-finite (see $[43,44])$. The key idea is to differentiate $Q(z, F(z))=0$ with respect to $z$; this shows that each derivative $\partial_{z}^{k} F(z)$ can be expressed as rational fraction in $z$ and $F(z)$, taking Taylor expansions of these fractions and using the relation $Q(z, F)=0$ allows then to write them as polynomials in $F$ of degree at most $d$, therefore they all live in the same space of dimension at most $d$, and thus it implies the claimed relation between the derivatives. It is amusing that this is in fact an old theorem rediscovered many times, by Tannery [110], Cockle and Harley [42, 68] in their method for solving quintic equations via ${ }_{4} F_{3}$ hypergeometric functions. Last but not least, this theorem can also be found in an unpublished manuscript of Abel [1, p. 287]!

The world of D-finite functions offers numerous closure properties; let us mention some of them related to algebraic functions:

Proposition 5 (Holonomy and closure properties for algebraic functions).

(1) $f$ and $1 / f$ are simultaneously $D$-finite if and only if $f^{\prime} / f$ is algebraic.

(2) $f$ and $\exp \left(\int f\right)$ are simultaneously $D$-finite if and only if $f^{\prime} / f$ is algebraic.

(3) Let $g$ be algebraic of genus $\geq 1$, then $f$ and $g(f)$ are simultaneously $D$-finite if and only if $f$ is algebraic.

(4) The Hadamard product of a rational and an algebraic function is algebraic.

(5) Let $\mathcal{A}$ be a context-free language and let $\mathcal{R}$ be a rational language, then one has a closure by intersection, Hadamard product, set difference: $\mathcal{A} \cap \mathcal{R}$, $\mathcal{A} \odot \mathcal{R}, \mathcal{A}-\mathcal{R}$, and $\mathcal{R}-\mathcal{A}$ are context-free (and thus have algebraic generating functions).

(6) Each algebraic function is the diagonal of a bivariate rational function.

(7) In finite fields, Hadamard products of algebraic functions are algebraic.

(8) The set of generalized hypergeometric functions ${ }_{n} F_{n-1}$ which are algebraic is well identified.

(9) It is possible to decide if a D-finite equation has algebraic solutions.

Proof. (1) is due to Harris \& Sibuya [69], (2) and (3) to Singer [104], (4) to Jungen [72]. (5) can be proven via the (pushdown) automata point of view (see also [22]). (6) is due to Denef and Lipshitz [48], (7) to Furstenberg [65], (8) to Schwarz [102] and Beukers \& Heckman [25]. It is still a challenge to find an efficient algorithm for (9), beyond the constructive approach given by Singer [103]. 
The linear recurrence satisfied by $f_{n}$ allows one to compute in linear time all the coefficients $f_{0}, \ldots, f_{n}$. More precisely, it is proved in [28] that there exists an algorithm of complexity $O\left(n d^{2} \ln d\right)$, where $d$ is the degree of the function. If one just wants the $n$-th coefficient $f_{n}$, it is possible to get it in $O(\sqrt{n})$ operations [40]. Many of these features (and a few others related to random generation and contextfree grammars, and corresponding asymptotics) are implemented in the "Algolib" library, a set of Maple packages developed by Flajolet, Salvy, Zimmermann, Chyzak, Mezzarobba,... (see http://algo.inria.fr/libraries/), see also the SageMath package by Kauers \& al. [86].

A less known fact is that these coefficients admit a closed form expression in terms of a finite linear combination of weighted multinomial numbers. The multinomial number is the number of ways to divide $m$ objects into $d$ groups, of cardinality $m_{1}, \ldots, m_{d}\left(\right.$ with $\left.m_{1}+\cdots+m_{d}=m\right)$ :

$$
\left[u_{1}^{m_{1}} \cdots u_{d}^{m_{d}}\right]\left(u_{1}+\cdots+u_{d}\right)^{m}=\left(\begin{array}{c}
m \\
m_{1}, \ldots, m_{d}
\end{array}\right)=\frac{m !}{m_{1} ! \ldots m_{d} !} .
$$

More precisely, one has the following theorem:

Theorem 1 (The Flajolet-Soria formula for coefficients of an algebraic function). Consider a power series implicitly defined by a polynomial equation $Q(z, f(z))=0$ (plus initial conditions for $f(z)$, in case the equation has several branches which are power series); therefore (up to a change of variable as explained in Proposition 2), $f(z)$ can equivalently be defined by $f(z)=P(z, f(z))$ where $P(z, y)$ is bivariate polynomial such that $[y] P \neq 1$ and $P(z, 0) \neq 0$. Then, the Taylor coefficients of $f(z)$ are given by the following finite sum

$$
f_{n}=\sum_{m \geq 1} \frac{1}{m}\left[z^{n} y^{m-1}\right] P^{m}(z, y) .
$$

Accordingly, applying the multinomial theorem on $P(z, y)=\sum_{i=1}^{d} a_{i} z^{b_{i}} y^{c_{i}}$ leads to

$$
f_{n}=\sum_{m \geq 1} \frac{1}{m} \sum_{\substack{m_{1}+\cdots+m_{d}=m \\
b_{1} m_{1}+\cdots+b_{d} m_{d}=n \\
c_{1} m_{1}+\cdots+c_{d} m_{d}=m-1}}\left(\begin{array}{c}
m \\
m_{1}, \ldots, m_{d}
\end{array}\right) a_{1}^{m_{1}} \ldots a_{d} m_{d},
$$

where all the $m_{i}$ 's are nonnegative integers; this sum is finite as it is more easily seen via the equivalent formula

$$
f_{n}=\sum_{m=0}^{n} \sum_{\substack{m_{1}+\cdots+m_{d}=m+1 \\ b_{1} m_{1}+\cdots+b_{d} m_{d}=n \\ c_{1} m_{1}+\cdots+c_{d} m_{d}=m}} m ! \frac{a_{1} m_{1}}{m_{1} !} \ldots \frac{a_{d} m_{d}}{m_{d} !} .
$$

Proof. We consider $y=P(z, y)$ as the perturbation at $u=1$ of the equation $y=u P(z, y)$, on which we apply (this is legitimate as $P(z, 0) \neq 0$ ) the Lagrange inversion formula (considering $u$ as the main variable, and $z$ as a fixed parameter); this gives: $\left[u^{m}\right] y=\frac{1}{m}\left[y^{m-1}\right] P(z, y)^{m}$. Then, summing for all $m$ (the sum is converging to $y(z)$, as it is well defined) and extracting $\left[z^{n}\right]$ on both sides leads to a non-trivial equality (and therefore to Equation (4)), because $[y] P(z, y) \neq 1$.

Note that Formula (4) still holds even if $P$ is not a polynomial, but more generally a power series $\in \mathbb{C}[[z, y]]$. 
This Flajolet-Soria formula (we coin the term) was first published in the habilitation thesis of Michèle Soria in 1990, and then in 1998 in the INRIA proceedings of the Algorithms Seminar, it is also mentioned in section VII.34 p. 495 in [63]; it has also been found by Gessel (as published in 1999 in the exercise 5.39 p. 148 of [107]), and it was finally also rediscovered in 2009 by Sokal [106]. It is worth pinpointing that Lagrange [80] initially presented his inversion formula in order to solve algebraic equations of any degree (considering the coefficients of the equations as parameters).

It is noteworthy that if $P$ has 3 terms or less, then the multiple sum in Formula 5 reduces to a single term, and it remains then just a simple sum on $m$. For instance, if we consider the equation

$$
f(z)=z+z^{2} f^{2}(z)+z^{3} f^{3}(z)
$$

we find for the coefficients the nice form (matter of taste!):

$$
f_{n+1}=\sum_{m=\left\lceil\frac{3}{5} n\right\rceil}^{\left\lfloor\frac{2}{3} n\right\rfloor} \frac{m !}{(n+1-m) !(5 m-3 n) !(2 n-3 m) !} .
$$

It is not possible to get this nice formula via a naive application of Lagrange's inversion formula, but it is a direct application of Theorem 1.

If one consider the case of the equation for the generating functions of $d$-ary trees, namely $y=1+z y^{d}$, then the formula simplifies a lot: each nested sum involves just one term; this gives the classical result $f_{n}=\frac{1}{(d-1) n+1}\left(\begin{array}{c}d n \\ n\end{array}\right)$.

More generally, the coefficients of an algebraic function defined by $y=P(z, y)$ are therefore given by $d-2$ nested sums of binomials, where $d$ is the number of terms of $P(z, y)$. Let $d_{1}$ be the $z$ degree of $P$ and let $d_{2}$ be the $y$ degree of $P$. The worst case number of nested sums in Equation (6) is therefore $\left(1+d_{1}\right)\left(1+d_{2}\right)-3$. E.g., if the $y$ and $z$ degree are bounded by 2, one will have 6 nested sums at most, as it is the case for $P(z, y)=1+y^{2}+z+z y+z y^{2}+z^{2}+z^{2} y+z^{2} y^{2}$, while $P(z, y)=1+z y^{2}+z^{2} y^{2}$ will lead to just one sum.

This can lead to impressive identities like several thousands of nested sums which actually simplify in a non trivial way to a single factorial-like product; an example of such a phenomena follows from the observation of Rodriguez-Villegas that

$$
F(z)=\sum_{n \geq 0} \frac{(30 n) ! n !}{(15 n) !(10 n) !(6 n) !} z^{n}
$$

is a (generalized hypergeometric) algebraic function of minimal degree 483840 . More generally, it is an interesting algorithmic question to get the minimal number of nested sums giving the coefficients $f_{n}$ (see [100] for an approach related to Karr's $\Pi \Sigma$-fields).

All those "closed forms" are nice for arithmetical/combinatorial properties, but they are not the right way to access to any type of universal asymptotics for the coefficients $f_{n}$; in the next section, we use a completely different approach to tackle these asymptotics questions. 


\section{Critical Exponents FOR COEFFicients of Algebraic FunCtion}

"It would at least be desirable to determine directly, from a positive (but reducible) system, the type of singular behaviour of the solution, but the systematic research involved in such a programme is yet to be carried out."

Quotation of Philippe Flajolet and Robert Sedgewick [63, p. 493]

In this section, we will characterize the singular behaviors of such systems, thus answering the wish of Flajolet \& Sedgewick. Our approach relies on the theory of Puiseux expansions which implies that the critical exponents are pure rational numbers for pure algebraic functions. (Pure algebraic means algebraic but not rational, pure rational means rational but not integer.) The full question is which subset of rational number do we get? We first start with the following proposition, which shows that all rational numbers are obtained if we do not constrain the algebraic function to satisfy a positive system of the type $\mathbf{y}=\mathbf{P}(z, \mathbf{y})$ :

Theorem 2. For any rational number $r$ that is not a negative integer, there exists an algebraic power series $F(z)=\sum_{n \geq 0} f_{n} z^{n}$ with positive integer coefficients $f_{n}$ which have critical exponent $r$, i.e. $f_{n} \sim C n^{r} A^{n}$ (for some positive constants $A, C$ ). What is more, this power series is $\mathbb{Z}$-algebraic.

Proof. First consider $F(z):=\frac{1-\left(1-a^{2} z\right)^{1 / a}}{a z}$, where $a$ is any positive or negative integer. Accordingly, its coefficients are given by $f_{n}=\left(\begin{array}{c}1 / a \\ n+1\end{array}\right) a^{2 n+1}(-1)^{n}$. The fact that the $f_{n}$ are positive integers was proven in [83], via a link with a variant of Stirling numbers. We give here another shorter proof. First, the Newton binomial theorem applied on $(1-a z F)^{a}=\left(1-a z^{2}\right)$ leads to an algebraic equation for $F(z)$ :

$$
F(z)=1+\sum_{k=2}^{a}\left(\begin{array}{l}
a \\
k
\end{array}\right) a^{k-2}(-1)^{k} z^{k-1}(F(z))^{k} .
$$

Then, if one sees this equation as a fixed point equation (as a rewriting rule in the style of context-free grammars), it is obvious that the $f_{n}$ 's belong to $\mathbb{Z}$. But as $f_{n+1}=a \frac{(a(n+1)-1) f_{n}}{n+2}$, it is also clear that the $f_{n}$ 's are indeed positive integers. Thus, we got any Puiseux critical exponent $1 / a$, and we now want to get any Puiseux critical exponent $b / a$, where $b$ is any positive integer (such that $b$ is not a multiple of $a$ ). For sure, it is not possible to take directly $F^{b}$ as it does not have Puiseux critical exponent $b / a$ (but $1 / a$ ), so we consider $G(z)=e(a z F(z)-1)^{b}$ (where $e=1$ if $a>b \bmod (2 a)$ and $e=-1$ elsewhere), which gives a series with integer coefficients (because of the integrality of the coefficients of $F$ ), positive coefficients (excepted a few of its first coefficients, for some monomials of degree less than $b$, as it comes from the Newton binomial expansion). Removing these negative coefficient terms gives a power series with only positive integer coefficients, with a Puiseux expansion of $G=e(-1)^{b}\left(1-a^{2} z\right)^{b / a}$ and consequently its coefficients have the asymptotics $C n^{-1-b / a} a^{-2 n}$ for some $C>0$.

One may then wonder if there is something stronger. For example, is it the case that for any radius of convergence, any critical exponent is possible? It happens not to be the case, as can be seen via a result of Fatou: a power series with integer coefficients and radius of convergence 1 is either rational or transcendental. However, one has the following neat generic behavior: 
Theorem 3 (Main result: dyadic critical exponents for $\mathbb{R}_{+}$-algebraic function). Any power series $F(z)=\sum_{n \geq 0} f_{n} z^{n}$ which is a solution of a well defined positive polynomial system of equations $\mathbf{y}=\mathbf{P}(z, \mathbf{y})$ (i.e. any $\mathbb{R}_{+}$-algebraic function, and a fortiori any $\mathbb{N}$-algebraic function) has the following asymptotic behavior:

- If $F(z)$ has a single singularity $\rho$ on its radius of convergence $|z|=\rho$ then we have

$$
f_{n} \sim C n^{\alpha} \rho^{-n}
$$

for some positive constant $C$ and a dyadic critical exponent $\alpha$ which belongs to the set

$$
\mathbb{D}_{2}=\left\{-1-\frac{1}{2^{k}}: k \geq 1\right\} \cup\left\{\frac{m}{2^{k}}-1: m \geq 1, k \geq 0\right\} .
$$

This set $\mathbb{D}_{2}$ of possible critical exponents is sparse on $[-3 / 2,-1)$ (starting with the values $\left.\left\{-\frac{3}{2},-\frac{5}{4},-\frac{9}{8},-\frac{17}{16}, \ldots\right\}\right)$ and dense on $(-1,+\infty)$, where it contains all dyadic numbers.

- If $F(z)$ has several singularities on its radius of convergence $|z|=\rho$ then there exists a integer $p \geq 1$ such that for every residue class $\ell \in\{0,1, \ldots, p-$ 1 ) we either have $f_{n}=0$ for sufficiently large $n$ with $n \equiv \ell \bmod p$ or

$$
f_{n} \sim C_{\ell} n^{\alpha_{\ell}} A_{\ell}^{n}, \quad n \equiv \ell \bmod p,
$$

where $A_{\ell}$ and $C_{\ell}$ are positive constants and the critical exponent $\alpha_{\ell}$ belongs to the set $\mathbb{D}_{2}$ defined in (9).

Proof. In the case of a single singularity on the radius of convergence, this theorem is the consequence of our stronger theorem on the Puiseux expansion of $\mathbb{R}_{+}$-algebraic functions (see Theorem 4 in Section 5.2). The periodic case (this essentially means that $F(z)$ has several dominant singularities, as may be seen from $F(z)=1 /(1-$ $\left.9 z^{2}\right)+1 /\left(1-8 z^{3}\right)$, which is 6 periodic) involves some additional care, and we consider the full details of such cases in Section 6 ; this even gives a proof which works in a more general setting than well defined polynomial systems (Theorem 6).

Accordingly, we have the following two propositions:

Proposition 6. All the critical exponents of Theorem 3 are indeed obtained, even for the subclass of $\mathbb{R}_{+}$-algebraic functions made of $\mathbb{N}$-algebraic functions.

Proof. By singularity analysis [63], there is a direct link between the singular behavior of $F(z)$ and the critical exponent of its coefficients $f_{n}$, namely: if $F(z) \sim$ $(1-A z)^{\alpha}$ then $f_{n} \sim \frac{1}{\Gamma(-\alpha)} n^{-1-\alpha} A^{n}$ (when $\rho=1 / A$ is the only singularity on the radius of convergence $|z|=\rho$ ). We will therefore show that all the singular behaviors corresponding to our dyadic set $\mathbb{D}_{2}$ of critical exponents are indeed obtained. The system of equations $y_{1}=z\left(y_{2}+y_{1}^{2}\right), y_{2}=z\left(y_{3}+y_{2}^{2}\right), y_{3}=z\left(1+y_{3}^{2}\right)$ has the following (explicit) solution

$$
\begin{aligned}
& y_{1}(z)=\frac{1-(1-2 z)^{1 / 8} \sqrt{2 z \sqrt{2 z \sqrt{1+2 z}+\sqrt{1-2 z}}+(1-2 z)^{3 / 4}}}{2 z} \\
& y_{2}(z)=\frac{1-(1-2 z)^{1 / 4} \sqrt{2 z \sqrt{1+2 z}+\sqrt{1-2 z}}}{2 z} \\
& y_{3}(z)=\frac{1-\sqrt{1-4 z^{2}}}{2 z}
\end{aligned}
$$


Here $y_{1}(z)$ has dominant singularity $(1-2 z)^{1 / 8}$ and it is clear that this example can be generalized: indeed, consider the system $y_{i}=z\left(y_{i+1}+y_{i}^{2}\right)$ for $i=1, \ldots, k-1$, and $y_{k}=z\left(1+y_{k}^{2}\right)$, it leads to behavior $(1-2 z)^{2^{-k}}$ for each $k \geq 1$. Now, taking the system of equations $y=z\left(y_{0}^{m}+y\right), y_{0}=z\left(1+2 y_{0} y_{1}\right)$ leads to a behavior $(1-2 z)^{-m 2^{-k}}$ for each $m \geq 1$ and $k \geq 0$. See also [75, 109] for another explicit combinatorial structure (a family of colored trees related to a critical composition) exhibiting all these critical exponents.

Proposition 7. Planar maps and several families of lattice paths (like Gessel walks) are not $\mathbb{N}$-algebraic (i.e., they cannot be generated by an unambiguous contextfree grammar).

Proof. This comes as a nice consequence of our Theorem 3: none of the families of planar maps of [11] can be generated by an unambiguous context-free grammar, because of their critical exponent $-5 / 2$. Also, the tables [29] of lattice paths in the quarter plane and their asymptotics (where some of the connection constants are guessed, but all the critical exponents are proved, and this is enough for our point) allow one to prove that many sets of jumps are giving a non-algebraic generating function, as they lead to a critical exponent which is a negative integer or involving $1 / 3$. One very neat example is Gessel walks (their algebraicity was a nice surprise [30]), where the hypergeometric formula for their coefficients leads to an asymptotic $4^{n} / n^{2 / 3}$ that is not compatible with $\mathbb{N}$-algebraicity. ${ }^{3}$

The critical exponents $-\frac{3}{4},-\frac{1}{4}, \frac{1}{4}$ which appear for walks on the slit plane [33] and other lattice paths questions [30] are compatible with $\mathbb{N}$-algebraicity, but these lattice paths are in fact not $\mathbb{N}$-algebraic (one can use Ogden's pumping lemma to prove that these walks can be not generated by a context-free grammar). To get a constructive method to decide $\mathbb{N}$-algebraicity (input: a polynomial equation, output: a context-free specification, whenever it exists) is a challenging task.

It is well-known that any $\mathbb{N}$-rational function has star height at most 2, e.g. the regular expression $\left(x\left(x\left(x x^{*}\right)^{*}\right)^{*}\right)^{*}$ involves 3 nested stars but can also be written as $1+x+x^{*}(3 x)^{*} x^{2}+x^{2} x^{*}$. For context-free grammars, one could consider the Chomsky and Greibach normal forms as a "similar flavor" result. On the other hand, one consequence of our Theorem 4 is that there exist context-free languages with unbounded "non-terminal height", more precisely:

Proposition 8. For all $k \in \mathbb{N}$, there exists a context-free language requiring inherently at least $k$ non-terminals for any grammar generating it and there exists a context-free language requiring inherently at least $k$ non strongly connected components for any grammar generating it.

Proof. Indeed, the integer $k$ (in Theorem 3) is the number of "nested dominant critical components" (as is transparent from our proof in Subsection 5.4), and each of these components requires at least one non-terminal. Multicolored super trees are an example of a structure requiring $k$ non-terminals.

In this section, we characterized the critical exponents of coefficients of algebraic functions; can we also characterize the subdominant critical exponents? Well, for

\footnotetext{
${ }^{3}$ The fact that critical exponents involving $1 / 3$ were not possible was an informal conjecture in the community for years. We thank Philippe Flajolet, Mireille Bousquet-Mélou and Gilles Schaeffer, who encouraged us to work on this question.
} 
algebraic functions, it is a consequence of their local Puiseux expansions and of singularity analysis that their coefficients behave e.g. like

$$
f_{n}=A^{n} n^{\alpha}\left(\sum_{k \geq 0} a_{k} \frac{1}{n^{k}}\right)+B^{n} n^{\beta}\left(\sum_{k \geq 0} b_{k} \frac{1}{n^{k}}\right)+O\left(C^{n}\right)
$$

(where $C<B$, with $A>B$ or $A=B$ and $\alpha>\beta$ ); what is more, we have proven in Theorem 3 that for $\mathbb{N}$-algebraic functions (and more generally for $\mathbb{Q}_{+}$-algebraic functions), $\alpha$ belongs to a specific subset of dyadic numbers. It is thus natural to ask what can be said on $\beta$ ? In fact, the union of Proposition 1 ( $\mathbb{Z}$-algebraic functions are the difference of two $\mathbb{N}$-algebraic functions) and Theorem 2 ( $\mathbb{Z}$-algebraic functions can have any rational critical exponent) implies that subdominant critical exponents $\beta$ of $\mathbb{N}$-algebraic functions can be any rational number which is not a negative integer.

\section{Finer asymptotics for $\mathbb{R}_{+}$-Algebraic functions}

The main goal of this section is to obtain a theorem on the Puiseux expansion of $\mathbb{R}_{+}$-algebraic functions.

The first subsection 5.1 gives precise conditions on the system. Subsection 5.2 gives our fundamental result, Theorem 4, which implies Theorem 3 of Section 4. Subsection 5.3 introduces the notion of dependency graph and a few preliminary lemmas, while the last subsection, Subsection 5.4, gives the proof of Theorem 4.

5.1. Well defined versus well posed systems of functional equations. In Definitions 3 and 4, we have described the so-called well posed and well defined systems of algebraic equations $\mathbf{y}=\mathbf{P}(z, \mathbf{y})$, and by definition it is clear that every positive well defined system is well posed, too. (One just has to start with $\mathbf{y}_{0}=\mathbf{0}$ and consider the iteration $\mathbf{y}_{k+1}=\mathbf{P}\left(z, \mathbf{y}_{k}\right)$ that converges formally and analytically to a tuple of power series $\left(f_{1}(z), \ldots f_{d}(z)\right)=\lim _{k \rightarrow \infty} \mathbf{y}_{k}$ with non-negative coefficients and the property $f_{i}(0)=0$.) However, as already indicated, there are also meaningful systems with power series solutions that are not well defined in the sense of Definition 4. The essential point is that such a meaningful system has power series solutions $y_{j}=f_{j}(z)$ with non-negative coefficients. (Of course, if the algebraic system is positive then we can expect non-negative coefficients, in particular if the iteration from above converges.)

Let us make this more precise by formulating an analytic condition for systems being meaningful.

Definition 5 (analytically well defined system). A positive system of polynomial equations $\mathbf{y}=\mathbf{P}(z, \mathbf{y})$ will be called analytically well defined if $\mathbf{P}(0, \mathbf{0})=\mathbf{0}$, if the Jacobian $\mathbf{P}_{\mathbf{y}}(0, \mathbf{0})=\left(\frac{\partial P_{i}}{\partial y_{j}}(0, \mathbf{0})\right)$ has spectral radius smaller than 1 , and if all solution functions $y_{j}=f_{j}(z)$ (with $f_{j}(0)=0$ ) are neither zero nor polynomials.

The condition on the spectral radius of $\mathbf{P}_{\mathbf{y}}$ ensures that the matrix $\mathbf{I}-\mathbf{P}_{\mathbf{y}}(0, \mathbf{0})$ (which is the Jacobian of the system $\mathbf{y}-\mathbf{P}(z, \mathbf{y})=\mathbf{0}$ ) is invertible so that the implicit function theorem implies that there is a unique tuple of analytic solution $\left(f_{1}(z), \ldots, f_{d}(z)\right)$ with $f_{j}(0)=0$. Furthermore this solution is obtained as the limit $\left(f_{1}(z), \ldots f_{d}(z)\right)=\lim _{k \rightarrow \infty} \mathbf{y}_{k}$ of the iteration $\mathbf{y}_{k+1}=\mathbf{P}\left(z, \mathbf{y}_{k}\right)$ with $\mathbf{y}_{0}=\mathbf{0}$ (actually the iteration is uniform for $|z| \leq \eta$, where $\eta>0$ is sufficiently small). Since all the iterates $\mathbf{y}_{k}$ are polynomials with non-negative coefficients, the (uniform) 
limit has the same non-negativity property. Note that this convergence need not be formal as the example $y=z+\frac{1}{2} y$ shows.

As mentioned above the condition $\mathbf{P}(0, \mathbf{0})=\mathbf{0}$ is not a real restriction. If $\mathbf{P}(0, \mathbf{0}) \neq \mathbf{0}$ and if there exists a non-negative vector $\mathbf{y}_{0}$ with $\mathbf{P}\left(0, \mathbf{y}_{0}\right)=\mathbf{y}_{0}$ such that the spectral radius of $\mathbf{P}_{\mathbf{y}}\left(0, \mathbf{y}_{0}\right)$ is smaller than 1 then the same argument as in the preceding paragraph shows that there exists a unique tuple of analytic solution $\left(f_{1}(z), \ldots, f_{d}(z)\right)$ with $\left(f_{1}(0), \ldots, f_{d}(0)\right)=\mathbf{y}_{0}$. Furthermore, by making a shift to reduce it to the case $\mathbf{P}(0, \mathbf{0})=\mathbf{0}$. We set $\tilde{\mathbf{y}}=\mathbf{y}+\mathbf{y}_{\mathbf{0}}$ so that we obtain a system for $\tilde{\mathbf{y}}$ of the form $\tilde{\mathbf{y}}=\tilde{\mathbf{P}}(z, \tilde{\mathbf{y}})$ with $\tilde{\mathbf{P}}(z, \tilde{\mathbf{y}})=\mathbf{P}\left(z, \tilde{\mathbf{y}}+\mathbf{y}_{0}\right)-\mathbf{y}_{0}$. Since $\mathbf{P}$ has non-negative coefficients, the same holds for $\tilde{\mathbf{P}}$. Consequently, there is no loss of generality to assume that we have a system $\mathbf{y}=\mathbf{P}(z, \mathbf{y})$ with $\mathbf{P}(0, \mathbf{0})=\mathbf{0}$.

Finally, it is very easy to detect, whether a meaningful system has some zero or polynomial solutions $f_{j}(z)$. Anyway, if zero or polynomial solutions $f_{j}(z)$ appear we just replace all appearances of $y_{j}$ by $f_{j}(z)$ and remove the $j$-th equation. This leads to a subsystem, where no solution $f_{j}(z)$ is zero or a polynomial. The Jacobian of the new (and smaller) system is just a submatrix of the original Jacobian and, thus, has a spectral radius that is not larger than that of the original one. So if the spectral radius of the Jacobian of the original system is smaller than 1, we get the same property for the new system.

Summing up it is no loss of generality to consider analytically well defined systems. Furthermore well defined systems are also analytically well defined.

Lemma 1. Every well defined system $\mathbf{y}=\mathbf{P}(z, \mathbf{y})$ is analytically well defined. And every analytically well defined system is well posed.

Proof. By definition a well defined system satisfies $\mathbf{P}(0, \mathbf{0})=\mathbf{0}$. Furthermore, the condition $\left[y_{j}\right] P_{i}\left(0,0, \ldots, y_{i}, \ldots\right)=0$ implies that $\mathbf{P}_{\mathbf{y}}(0, \mathbf{0})$ is the zero matrix which has spectral radius 0 . Finally the terminating condition ensures that there are no zero or polynomial solutions $f_{j}(z)$.

We already discussed that every analytically well defined system has a tuple of power series solutions $f_{j}(z)$ with non-negative coefficients. This completes the proof of the lemma.

There are several reasons why we distinguish between well defined and analytically well defined systems of equations. From a formal point of view well defined systems are very easy to describe, since we just have to look at the polynomial system. On the other hand it excludes some meaningful systems (in particular systems having epsilon production or loops of monic productions with a total weight $<1)$. When it comes to proofs, it is easier to work with analytic conditions like the condition on the spectral radius, and therefore we will mainly rely on analytically well defined systems. A good motivation for this approach is that the analysis of the behavior of the spectral radius $r\left(\mathbf{P}_{\mathbf{y}}(z, \mathbf{f}(z))\right)$ actually plays a dominant rôle in the proof of Theorem 4 .

5.2. Critical exponents of $\mathbb{R}_{+}$-algebraic functions. Our first main goal is to characterize the Puiseux critical exponents of the singular expansions of $f_{j}(z)$ at the radius of convergence $\rho_{j}$, when we are considering power series solutions of analytically well defined positive polynomial systems of equations. The main observation is that these exponents are special dyadic rational numbers - in contrast to general algebraic functions (see Theorem 2). 
Theorem 4. Let $\mathbf{y}=\mathbf{P}(z, \mathbf{y})$ be an analytically well defined positive polynomial system of functional equations.

Then the solutions $f_{j}(z)$ have positive and finite radii of convergence $\rho_{j}$ and the Puiseux critical exponents are either of the form $2^{-k_{j}}$ for some integer $k_{j} \geq 1$ or of the form $-m_{j} 2^{-k_{j}}$ for some integer $m_{j} \geq 1$ and $k_{j} \geq 0$. In particular the singular behavior of $f_{j}(z)$ around $\rho_{j}$ is either of type

$$
f_{j}(z)=f_{j}\left(\rho_{j}\right)+c_{j}\left(1-z / \rho_{j}\right)^{2^{-k_{j}}}+c_{j}^{\prime}\left(1-z / \rho_{j}\right)^{2 \cdot 2^{-k_{j}}}+\cdots,
$$

where $c_{j} \neq 0$ (and an integer $k_{j} \geq 1$ ) or of type

$$
f_{j}(z)=\frac{d_{j}}{\left(1-z / \rho_{j}\right)^{m_{j} 2^{-k_{j}}}}+\frac{d_{j}^{\prime}}{\left(1-z / \rho_{j}\right)^{\left(m_{j}-1\right) 2^{-k_{j}}}}+\cdots,
$$

where $d_{j} \neq 0$ (and integers $m_{j} \geq 1$ and $k_{j} \geq 0$ ).

This theorem gives already a partial result on the asymptotic structure of the coefficients $f_{j ; n}$ of $f_{j}(z)$. If we assume that $\rho_{j}$ is the only singularity on the circle of convergence $|z|=\rho_{j}$ (which we call the aperiodic case) then the transfer theorem of Flajolet and Odlyzko [62] implies that $f_{j ; n}$ is asymptotically given by

$$
f_{j ; n} \sim C_{j} n^{\alpha_{j}} \rho_{j}^{-n} \quad(n \rightarrow \infty),
$$

where $C_{j}>0, \rho_{j}>0$, and $\alpha_{j}$ is either of the form $\alpha_{j}=-2^{-k_{j}}-1$ for some integer $k_{j} \geq 1$ or of the form $\alpha_{j}=m_{j} / 2^{k_{j}}-1$ for some integers $k_{j} \geq 0$ and $m_{j} \geq 1$.

Actually we will provide a complete answer to the problem in the periodic case too; see Theorems 5 and 6 . In all cases we obtain asymptotic properties as stated in (12); however, we have to distinguish between residue classes modulo some positive integer $p$, and the asymptotic scale might be different in each residue class. In order to make the presentation more transparent we deal first with Theorem 4 and then consider the more involved question of periodicities.

5.3. Dependency graph and auxiliary results. A main ingredient of the proof of Theorem 4 is the analysis of the dependency graph $G$ of the system $y_{j}=$ $P_{j}\left(z, y_{1}, \ldots, y_{d}\right), 1 \leq j \leq d$. The vertex set is $\{1, \ldots, d\}$ and there is a directed edge from $i$ to $j$ if $P_{j}$ depends on $y_{i}$ (see Figure 2). If the dependency graph is strongly connected then we are in a very special case of Theorem 4, for which one has one of the following two situations (see [52]):

Lemma 2 (rational singular behavior). Let $\mathbf{y}=\mathbf{A}(z) \mathbf{y}+\mathbf{B}(z)$ be an affine analytically well defined system of equations, where the dependency graph is strongly connected. Then the functions $f_{j}(z)$ have a joint polar singularity $\rho$ of order one as the dominant singularity:

$$
f_{j}(z)=\frac{c_{j}(z)}{1-z / \rho},
$$

where $c_{j}(z)$ is non-zero and analytic at $z=\rho$.

Proof. In the affine case the Jacobian of the system equals $\mathbf{A}(z)$. Hence by assumption the spectral radius of $\mathbf{A}(0)$ is smaller than 1 which implies that $\mathbf{f}(z)$ can be represented as

$$
\mathbf{f}(z)=(\mathbf{I}-\mathbf{A}(z))^{-1} \mathbf{B}(z)
$$

if $|z|$ is sufficiently small. Since the dependency graph is strongly connected it follows that the matrix $\mathbf{A}(z)$ is a positive and irreducible matrix if $z>0$. Consequently 
by Perron-Frobenius theory the spectral radius $r(\mathbf{A}(z))$ is a strictly increasing and continuous function in $z>0$. Hence there exists a unique $\rho>0$ with $r(\mathbf{A}(\rho))=1$. Again by Perron-Frobenius theory the spectral radius is the dominant eigenvalue of $\mathbf{A}(\rho)$ that is simple, too. This also implies that the function

$$
z \mapsto \operatorname{det}(\mathbf{I}-\mathbf{A}(z))
$$

has a simple root at $z=\rho$. Of course this leads to a simple polar singularity for $\mathbf{f}(z)$. Note that this singularity has to appear for all functions $f_{j}(z), 1 \leq j \leq d$, since the system is strongly connected.

Lemma 3 (algebraic singular behavior). Let $\mathbf{y}=\mathbf{P}(z, \mathbf{y})$ be an affine analytically well defined polynomial system of equations that is not affine and where the dependency graph is strongly connected. Then the functions $f_{j}(z)$ have a joint radius of convergence $\rho$ and Puiseux singular exponent $1 / 2$ at $z=\rho$, that is, they can be locally represented as

$$
f_{j}(z)=g_{j}(z)-h_{j}(z) \sqrt{1-\frac{z}{\rho}}
$$

where $g_{j}(z)$ and $h_{j}(z)$ are non-zero and analytic at $z=\rho$.

Proof. ${ }^{4}$ Since the system is positive and well posed, there exists a unique solution $\mathbf{f}(z)$ with $\mathbf{f}(0)=\mathbf{0}$ which has non-negative coefficients. By assumption the spectral radius of the Jacobian $\mathbf{P}_{\mathbf{y}}(0, \mathbf{0})$ is smaller than 1 . Since the dependency graph is strongly connected the matrix $\mathbf{P}_{\mathbf{y}}(z, \mathbf{f}(z))$ is a positive and irreducible matrix for $z>0$ (as long as $\mathbf{f}(z)$ is regular). Furthermore the spectral radius $\left.r\left(\mathbf{P}_{\mathbf{y}}(z, \mathbf{f}(z))\right)\right)$ is a strictly increasing and continuous function in $z>0$. Recall that (by the implicit function theorem) $\mathbf{f}(z)$ is certainly regular if $\left.r\left(\mathbf{P}_{\mathbf{y}}(z, \mathbf{f}(z))\right)\right)<1$. Hence it follows that there exists $\rho>0$ with the property that $\mathbf{f}(\rho)$ exists (although it will not be a regular point) and $\left.r\left(\mathbf{P}_{\mathbf{y}}(\rho, \mathbf{f}(\rho))\right)\right)=1$. Thus, we can now apply the DrmotaLalley-Woods theorem $[53,63]$ which implies that $\rho$ is the dominant singularity for $f_{j}(z)$ and they are all of square-root type.

In the proof of Theorem 4 we will use extended versions of Lemmas 2 and 3, where we introduce additional parameters, that is, we consider systems of functional equations of the form

$$
\mathbf{y}=\mathbf{P}(z, \mathbf{y}, \mathbf{u}),
$$

where $\mathbf{P}$ is now a polynomial in $z, \mathbf{y}, \mathbf{u}$ with $\mathbf{P}(0, \mathbf{0}, \mathbf{0})=\mathbf{0}$ and non-negative coefficients and where the dependency graph (with respect to $\mathbf{y}$ ) is strongly connected. We also assume that $r\left(\mathbf{P}_{\mathbf{y}}(0, \mathbf{0}, \mathbf{0})\right)<1$ so that we can consider the solution that we denote by $\mathbf{f}(z, \mathbf{u})$. We also consider situations where $\mathbf{u}$ is strictly positive from the very beginning. In this case we restrict ourselves to situations where $\mathbf{f}(0, \mathbf{u})$ exists and where the spectral radius of the Jacobian $\mathbf{P}_{\mathbf{y}}(0, \mathbf{f}(0, \mathbf{u}), \mathbf{u})$ is smaller than 1.

If we are in the affine setting $(\mathbf{y}=\mathbf{A}(z, \mathbf{u}) \mathbf{y}+\mathbf{B}(z, \mathbf{u}))$ it follows that $\mathbf{f}(z, \mathbf{u})$ has a polar singularity:

$$
f_{j}(z, \mathbf{u})=\frac{c_{j}(z, \mathbf{u})}{1-z / \rho(\mathbf{u})}
$$

\footnotetext{
${ }^{4}$ The proofs of Lemma 2 and 3 could be simplified, since we work just with algebraic functions. However, in Section 9 we will also consider entire systems of functional equations and the present proof generalizes to this situation.
} 
where the functions $\rho(\mathbf{u})$ and $c_{j}(z, \mathbf{u})$ are non-zero and analytic (see Lemma 4). Please observe that we have to distinguish two cases. If $\mathbf{A}(z, \mathbf{u})=\mathbf{A}(z)$ does not depend on $\mathbf{u}$ then $\rho(\mathbf{u})=\rho$ is constant and the dependency from $\mathbf{u}$ just comes from $\mathbf{B}(z, \mathbf{u})$. Of course, if $\mathbf{A}(z, \mathbf{u})$ depends on $\mathbf{u}$ then $\rho(\mathbf{u})$ is not constant. More precisely it depends exactly on those coordinates of $\mathbf{u}$ that appear in $\mathbf{A}(z, \mathbf{u})$.

Similarly, in the non-affine setting we obtain representations of the form

$$
f_{j}(z, \mathbf{u})=g_{j}(z, \mathbf{u})-h_{j}(z, \mathbf{u}) \sqrt{1-\frac{z}{\rho(\mathbf{u})}},
$$

where the functions $\rho(\mathbf{u}), g_{j}(z, \mathbf{u})$, and $h_{j}(z, \mathbf{u})$ are non-zero and analytic. In this case $\rho(\mathbf{u})$ is always non-constant and depends on all coordinates of $\mathbf{u}$ (see Lemma 4).

Actually we have to be careful with the property that $\rho(\mathbf{u})$ is analytic. By looking at the above proofs it immediately follows that $\rho(\mathbf{u})$ exists but analyticity is not immediate. For notational convenience we will denote by $D_{0}$ the set of positive real vectors $\mathbf{u}$ for which $r\left(\mathbf{P}_{\mathbf{y}}(0, \mathbf{f}(0, \mathbf{u}), \mathbf{u})\right)<1$.

Lemma 4. The function $\rho(\mathbf{u})$ that appears in the representations (13) and (14) is analytic in a proper complex neighborhood of $D_{0}$. Moreover, if $\mathbf{u} \in D_{0}$ is real then $\rho(\mathbf{u})$ tends to 0 when $\mathbf{u}$ approaches the boundary of $D_{0}$ in a way that all coordinates of $\mathbf{u}$ are non-decreasing.

Proof. We recall a general method for reducing the system $\mathbf{y}=\mathbf{P}(z, \mathbf{y}, \mathbf{u})$ to a single equation if the dependency graph is strongly connected. We split between the first equation $y_{1}=P_{1}\left(z, y_{1}, \overline{\mathbf{y}}, \mathbf{u}\right)$ and $\overline{\mathbf{y}}=\overline{\mathbf{P}}\left(z, y_{1}, \overline{\mathbf{y}}, \mathbf{u}\right)$, where $\overline{\mathbf{y}}=\left(y_{2}, \ldots, y_{d}\right)$ and $\overline{\mathbf{P}}=\left(P_{2}, \ldots, P_{d}\right)$. Suppose that $r\left(\mathbf{P}_{\mathbf{y}}\left(\rho, \mathbf{y}_{0}, \mathbf{u}_{0}\right)\right)=1$ for some $\mathbf{u}_{0} \in D_{0}$ (and $\left.\rho=\rho\left(\mathbf{u}_{0}\right), \mathbf{y}_{0}=\mathbf{y}_{0}\left(\mathbf{u}_{0}\right)\right)$ then by Perron-Frobenius theory $r\left(\overline{\mathbf{P}}_{\overline{\mathbf{y}}}\left(\rho, y_{1,0}, \overline{\mathbf{y}}_{0}, \mathbf{u}_{0}\right)\right)<1$ since $\overline{\mathbf{P}}_{\overline{\mathbf{y}}}$ is the submatrix that results from $\mathbf{P}_{\mathbf{y}}$ by deleting the first row and column. Hence by the implicit function theorem there is an analytic solution $\overline{\mathbf{f}}\left(z, y_{1}, \mathbf{u}\right)$ of the subsystem (locally around $\rho, y_{1,0}, \mathbf{u}_{0}$ ) that we can insert into the first equation so that we are left with a single equation:

$$
y_{1}=P_{1}\left(z, y_{1}, \overline{\mathbf{f}}\left(z, y_{1}, \mathbf{u}\right), \mathbf{u}\right)=Q\left(z, y_{1}, \mathbf{u}\right) .
$$

In the affine case this rewrites to $y_{1}=a(z, \mathbf{u}) y_{1}+b(z, \mathbf{u})$ or $y_{1}=b(z, \mathbf{u}) /(1-a(z, \mathbf{u}))$. Since we are in the well posed case, $a(z, \mathbf{u})$ depends on $z$ and $\mathbf{u}$. Furthermore $a\left(\rho\left(\mathbf{u}_{0}, \mathbf{u}_{0}\right)\right)=1$. Since we certainly have $a_{z}\left(\rho\left(\mathbf{u}_{0}\right), \mathbf{u}_{0}\right)>0$ the implicit function theorem implies that $\rho(\mathbf{u})$ is analytic locally at $\mathbf{u}_{0} \in D_{0}$.

In the non-affine case, the situation is similar but slightly more involved. Since the equation $y_{1}=Q\left(z, y_{1}, \mathbf{u}\right)$ is singular for $z=\rho, y_{1}=y_{1,0}, \mathbf{u}=\mathbf{u}_{0}$ we have:

$$
y_{1,0}=Q\left(\rho, y_{1,0}, \mathbf{u}_{0}\right), \quad 1=Q_{y_{1}}\left(\rho, y_{1,0}, \mathbf{u}_{0}\right) .
$$

Furthermore this small system can be used to calculate $\rho(\mathbf{u})$ (locally around $\mathbf{u}_{0}$. Here $\mathbf{u}$ is the variable and $\rho=\rho(\mathbf{u}), y_{1,0}=y_{1,0}\left(\mathbf{u}_{0}\right)$ are the unknown functions. By the implicit function theorem we just have to observe that the corresponding Jacobian

$$
\left(\begin{array}{cc}
Q_{z} & Q_{y_{1}}-1 \\
Q_{z y_{1}} & Q_{y_{1} y_{1}}
\end{array}\right)=\left(\begin{array}{cc}
Q_{z} & 0 \\
Q_{z y_{1}} & Q_{y_{1} y_{1}}
\end{array}\right)
$$

is regular. We certainly have $Q_{z}>0$ and $Q_{y_{1} y_{1}}>0$ (if the system is non-affine). Hence the determinant of the Jacobian is non-zero, which implies that $\rho(\mathbf{u})$ is analytic in a complex neighborhood of $\mathbf{u}_{0} \in D_{0}$. 
Finally if $\mathbf{u}$ increases and gets close to the boundary of $D_{0}$ then the spectral radius $r\left(\mathbf{P}_{\mathbf{y}}(0, \mathbf{f}(0, \mathbf{u}), \mathbf{u})\right)$ is close to 1 . This implies that the radius of convergence $\rho(\mathbf{u})$ has to be close to zero.

5.4. Proof of our Theorem 4 on the possible Puiseux expansions. In order to give a flavor of the proof of Theorem 4 in the general case, we first discuss a simple example. Suppose that we are dealing with the system of equations depicted in Figure 2. The first step is to consider the reduced dependency graph $\widetilde{G}$ which is obtained by the following procedure. The vertices of $\widetilde{G}$ are the strongly connected components of $G$, these are the maximal strongly connected subgraphs. In our example, these components are $\{1\},\{2\},\{3,4\},\{5,6\}$. Next, two different components $C_{1}, C_{2}$ in $\widetilde{G}$ are linked by a directed edge if there exist vertices $v_{1} \in C_{1}$ and $v_{2} \in C_{2}$ that are linked in $G$. The resulting graph $\widetilde{G}$ (that is also depicted in Figure 2) is acyclic and comprises precisely the connectivity relation in $G$. Furthermore, this directed acyclic graph (DAG) $\widetilde{G}$ indicates how the system of equations $y_{j}=P_{j}\left(z, y_{1}, \ldots, y_{d}\right)$ can be solved. First, one considers all components in $G$ (vertices in $\widetilde{G}$ ) with zero in-degree. (Since $\widetilde{G}$ is acyclic such vertices have to exist.) In our example, these are the components $\{3,4\}$ and $\{5,6\}$ which correspond to the subsystems

$$
\begin{aligned}
& y_{3}=P_{3}\left(z, y_{3}, y_{4}\right) \\
& y_{4}=P_{4}\left(z, y_{3}, y_{4}\right)
\end{aligned} \quad \text { and } \quad \begin{aligned}
& y_{5}=P_{3}\left(z, y_{5}, y_{6}\right) \\
& y_{6}=P_{4}\left(z, y_{5}, y_{6}\right)
\end{aligned}
$$

These subsystems can be independently solved and their solutions $f_{3}(z), f_{4}(z)$ and $f_{5}(z), f_{6}(z)$, respectively, can be put into the remaining equations:

$$
\begin{aligned}
& y_{1}=P_{1}\left(z, y_{1}, y_{2}, f_{5}(z)\right) \\
& y_{2}=P_{2}\left(z, y_{2}, f_{3}(z), f_{5}(z)\right) .
\end{aligned}
$$

This resulting system of equation for the unknown $y_{1}=f_{1}(z), y_{2}=f_{2}(z)$ corresponds to a dependency graph, where the corresponding vertices 3,4 and 5,6 are deleted. This is depicted in Figure 2.

As above we can solve all equations that correspond to components in the reduced dependency graph with zero in-degree. In our example this is only the component $\{2\}$. With this solution $f_{2}(z)$ (where we already use the previous solutions $f_{3}(z)$, $\left.f_{5}(z)\right)$ we can finally obtain $y_{1}=f_{1}(z)$ by solving the remaining equation

$$
y_{1}=P_{1}\left(z, y_{1}, f_{2}(z), f_{5}(z)\right) \text {. }
$$

Of course, this procedure generalizes easily to any system of functional equations of the form $y_{j}=P_{j}\left(z, y_{1}, \ldots, y_{d}\right)$.

Actually we will do a two-step procedure. First, for each component of the dependency graph we solve the corresponding system, where the input functions are considered as additional parameters $\mathbf{u}$ (as discussed in Section 5.3). For example, for the component $\{2\}$, one should consider the solution $f_{2}\left(z ; y_{3}, y_{5}\right)$.

According to the generalizations of Lemma 2 and Lemma 3 these functions have either a polar singularity or a square-root singularity $\rho(\mathbf{u})$ (that depends on the parameters). In the second step we then insert step by step the solutions of the subsystems and get the solution of the system. The main problem is to trace the leading singularity. For example, in the first example in the proof of Proposition 6, the square-root singularities coalesce and give rise to singularities of fourth and 


$$
\left\{\begin{array}{l}
y_{1}=P_{1}\left(z, y_{1}, y_{2}, y_{5}\right) \\
y_{2}=P_{2}\left(z, y_{2}, y_{3}, y_{5}\right) \\
y_{3}=P_{3}\left(z, y_{3}, y_{4}\right) \\
y_{4}=P_{4}\left(z, y_{3}, y_{4}\right) \\
y_{5}=P_{5}\left(z, y_{5}, y_{6}\right) \\
y_{6}=P_{6}\left(z, y_{5}, y_{6}\right) .
\end{array}\right.
$$
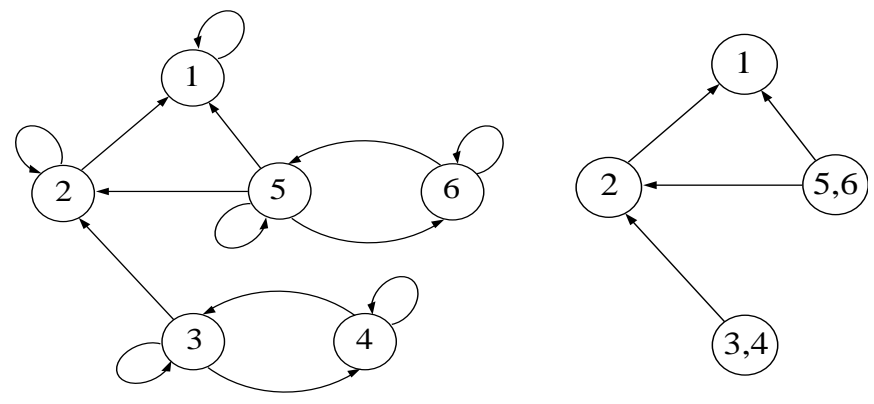

Figure 2. A positive system, its dependency graph $G$ and its reduced dependency graph $\widetilde{G}$. None of these graphs are here strongly connected: e.g. the state 1 is a sink; it is thus a typical example of a system not covered by the Drmota-Lalley-Woods theorem, but covered by our new result implying dyadic critical exponents.

eighth roots. Similarly, in the second example in the proof of Proposition 6 , a polar and a square-root singularity give rise to a singularity of the form $1 / \sqrt{1-2 z}$.

The main problem in the proof of Theorem 4 is to show that this insertion process does not create other singularities than stated.

We fix some notation. Let $G$ denote the dependency graph of the system and $\widetilde{G}$ the reduced dependency graph. Its vertices are the strongly connected components $C_{1}, \ldots C_{L}$ of $G$. We can then reduce the dependency graph to its components (see Figure 2). Let $\mathbf{y}_{1}, \ldots, \mathbf{y}_{L}$ denote the system of vectors with coordinates corresponding to the components $C_{1}, \ldots C_{L}$ and let $\mathbf{u}_{1}, \ldots, \mathbf{u}_{L}$ denote the input vectors related to these components. In the above example we have $C_{1}=\{1\}, C_{2}=\{2\}$, $C_{3}=\{3,4\}, C_{4}=\{5,6\}, \mathbf{y}_{1}=y_{1}, \mathbf{y}_{2}=y_{2}, \mathbf{y}_{3}=\left(y_{3}, y_{4}\right), \mathbf{y}_{4}=\left(y_{5}, y_{6}\right)$, and $\mathbf{u}_{1}=\left(y_{2}, y_{5}\right), \mathbf{u}_{2}=\left(y_{3}, y_{5}\right), \mathbf{u}_{3}=\emptyset, \mathbf{u}_{4}=\emptyset$.

As mentioned above in the first step, for each strongly connected component $C_{\ell}$ we solve the corresponding subsystem in the variables $z$ and $\mathbf{u}_{\ell}$ and obtain solutions $\mathbf{f}\left(z, \mathbf{u}_{\ell}\right), 1 \leq \ell \leq L$. In our example these are the functions $\mathbf{f}_{1}\left(z, \mathbf{u}_{1}\right)=f_{1}\left(z ; y_{2}, y_{5}\right)$, $\mathbf{f}_{2}\left(z, \mathbf{u}_{2}\right)=f_{2}\left(z ; y_{3}, y_{5}\right), \mathbf{f}_{3}\left(z, \mathbf{u}_{3}\right)=\left(f_{3}(z), f_{4}(z)\right), \mathbf{f}_{4}\left(z, \mathbf{u}_{4}\right)=\left(f_{5}(z), f_{6}(z)\right)$.

Finally, for each component $C_{\ell}$ we define the set $D_{\ell}$ of real vectors $\mathbf{u}_{\ell}$ for which the spectral radius of the Jacobian of the $\ell$-th subsystem evaluated at $z=0$, $\mathbf{y}_{\ell}=f_{\ell}\left(0, \mathbf{u}_{\ell}\right.$ is smaller than 1.

Since the dependency graph $\widetilde{G}$ is acyclic there are components $C_{\ell_{1}}, \ldots, C_{\ell_{m}}$ with no input, that is, the corresponding functions $\mathbf{f}_{\ell_{1}}(z), \ldots, \mathbf{f}_{\ell_{m}}(z)$ can be computed without any further information. By Lemmas 2 and 3 they either have a polar singularity or a square-root singularity, that is, they are of the types that are included in the statement of Theorem 4.

Now we proceed inductively. We consider a strongly connected component $C_{\ell}$ together with its function $\mathbf{f}_{\ell}\left(z, \mathbf{u}_{\ell}\right)$ and assume that all the functions $f_{j}(z)$ that correspond to the input coordinates $\mathbf{u}_{\ell}$ are already known and that their leading singularities are of the two types stated in Theorem 4. By the discussion following Lemma 2 and 3 it follows that coordinate functions in $\mathbf{f}_{\ell}\left(z, \mathbf{u}_{\ell}\right)$ have either a common polar singularity or a common square-root singularity $\rho\left(\mathbf{u}_{\ell}\right)$. We distinguish between three cases: 
(1) First let us assume that $\mathbf{f}_{\ell}\left(z, \mathbf{u}_{\ell}\right)$ comes from an affine system and, thus, has a polar singularity. Since all functions in $\mathbf{f}_{\ell}\left(z, \mathbf{u}_{\ell}\right)$ have the same form we just consider one of these functions and denote it by $f\left(z, \mathbf{u}_{\ell}\right)$ :

$$
f\left(z, \mathbf{u}_{\ell}\right)=\frac{c\left(z, \mathbf{u}_{\ell}\right)}{1-z / \rho\left(\mathbf{u}_{\ell}\right)} .
$$

If $\rho\left(\mathbf{u}_{\ell}\right)=\rho^{\prime}$ is constant then the only dependency from $\mathbf{u}_{\ell}$ comes from the numerator $c\left(z, \mathbf{u}_{\ell}\right)$. Since this solution comes from an affine system, $c\left(z, \mathbf{u}_{\ell}\right)$ is just a linear combination of the polynomials of $\mathbf{B}\left(z, \mathbf{u}_{\ell}\right)$ with coefficient functions that depend only on $z$ (this follows from the expansion of $(\mathbf{I}-\mathbf{A}(z))^{-1} \mathbf{B}\left(z, \mathbf{u}_{\ell}\right)$ ). Furthermore, since $f\left(z, \mathbf{u}_{\ell}\right)$ is (in principle) a power series in $z$ and $\mathbf{u}_{\ell}$ with non-negative coefficients the coefficients of this polynomial (if $z$ is some positive real number) have to be non-negative, too.

When we substitute $\mathbf{u}_{\ell}$ by the functions $f_{j}(z)$ that correspond to $\mathbf{u}_{\ell}$, we obtain the functions $f(z)$ that correspond to the component $C_{\ell}$. We have to consider the following subcases:

(1.1) The dominating singularities $\rho_{j}$ of the $f_{j}(z)$ 's are larger than $\rho^{\prime}$ :

In this case the resulting dominating singularity $\rho_{\ell}$ is $\rho^{\prime}$ and we just get a polar singularity for $f(z)$.

(1.2) At least one of the dominating singularities $\rho_{j}$ of the functions $f_{j}(z)$ is smaller than $\rho^{\prime}$ :

Let $\rho^{\prime \prime}$ denote the smallest of these singularities. If all of the functions $f_{j}(z)$ with $\rho_{j}=\rho^{\prime \prime}$ have a singular behavior of the form (10) then we just make a local expansion of $c\left(z, \mathbf{u}_{\ell}\right)$ at the corresponding points $f_{j}\left(\rho^{\prime \prime}\right)$ (for $\left.u_{j}\right)$ and observe again an expansion of this form. Note the largest appearing $k_{j}$ reappears in the expansion of $f(z)$.

Second, if at least one of the functions $f_{j}(z)$ with $\rho_{j}=\rho^{\prime \prime}$ is of type (11) then we use the property that $c\left(z, \mathbf{u}_{\ell}\right)$ is just a polynomial in $\mathbf{u}_{\ell}$ (with non-negative coefficients). It is clear that the leading singular behavior comes from these functions; actually they have to be multiplied and added. However, since functions of the type (11) are closed under multiplication and addition, this gives again a function of type (11). Note that the coefficient functions that depend just on $z$ have to be expanded at $\rho^{\prime \prime}$, too, and do not disturb the overall structure.

(1.3) The smallest dominating singularities $\rho_{j}$ of the functions $f_{j}(z)$ equals $\rho^{\prime}$ :

Here we can argue similarly to the previous case. If all of the functions $f_{j}(z)$ with $\rho_{j}=\rho^{\prime}$ have a singular behavior of the form (10) then we perform a local expansion in the numerator. Let $\tilde{k}$ be the largest $k_{j}$ that appears. Then we interpret the polar singularity $\left(1-z / \rho^{\prime}\right)^{-1}$ as $\left(1-z / \rho^{\prime}\right)^{-m 2^{-\tilde{k}}}$ with $m=2^{\tilde{k}}$ and obtain a singular expansion of the form (11). If at least one of the functions $f_{j}(z)$ with $\rho_{j}=\rho^{\prime}$ is of type (11) then we use the polynomial structure of the numerator as above and obtain an expansion of the form (11). By combining this with the factor $\left(1-z / \rho^{\prime}\right)^{-1}$ we finally obtain an expansion of the form (11) for $f(z)$, too.

(2) Second let us (again) assume that $\mathbf{f}_{\ell}\left(z, \mathbf{u}_{\ell}\right)$ comes from an affine system (and has a polar singularity) of the form (17); however, we now assume that $\rho\left(\mathbf{u}_{\ell}\right)$ is not constant but depends on some of the $u_{j}$ (not necessarily on all of them). In this case we study first the behavior of the denominator when $\mathbf{u}_{j}$ is replaced by 
the corresponding functions $f_{j}(z)$. For the sake of simplicity we will work with the difference $\rho\left(\mathbf{u}_{j}\right)-z$. Of course this is equivalent to the discussion of the denominator $1-z / \rho\left(\mathbf{u}_{j}\right)$, since the factor $\rho\left(\mathbf{u}_{j}\right)$ can be also put to the numerator. Finally let $J_{\ell}^{\prime}$ denote the set of indices of functions $u_{j}$ for which the function $\rho\left(\mathbf{u}_{\ell}\right)$ really depends on.

Let $\rho^{\prime}$ denote the smallest radius of convergence of the functions $f_{j}(z), j \in J_{\ell}^{\prime}$. Then we consider the difference $\delta(z)=\rho\left(\left(f_{j}(z)\right)_{j \in J_{\ell}^{\prime}}\right)-z$. We have to consider the following subcases for the denominator:

(2.1) $\delta\left(\rho^{\prime \prime}\right)=0$ for some $\rho^{\prime \prime}<\rho^{\prime}$ such that $\left(f_{j}\left(\rho^{\prime \prime}\right)\right)_{j \in J_{\ell}^{\prime}} \in D_{\ell}$ :

First we note that $\delta(z)$ has at most one positive zero since $\rho\left(\left(f_{j}(z)\right)_{j \in J_{\ell}^{\prime}}\right)$ is decreasing and $z$ is increasing. Furthermore the derivative satisfies $\delta^{\prime}\left(\rho^{\prime \prime}\right)>0$. Consequently we have a simple zero $\rho^{\prime \prime}$ in the denominator.

(2.2) We have $\delta\left(\rho^{\prime}\right)=0$ such that $\left(f_{j}\left(\rho^{\prime}\right)\right)_{j \in J_{\ell}^{\prime}} \in D_{\ell}$ :

In this case all functions $f_{j}(z), j \in J_{\ell}^{\prime}$, with $\rho_{j}=\rho^{\prime}$ have to be of type (10). Consequently $\delta(z)$ behaves like

$$
c\left(1-z / \rho^{\prime}\right)^{2^{-\tilde{k}}}+\ldots,
$$

where $c>0$ and $\tilde{k}$ is the largest appearing $k_{j}$ (among those functions $f_{j}(z)$ with $\left.\rho_{j}=\rho^{\prime}\right)$.

(2.3) We have $\delta\left(\rho^{\prime}\right)>0$ such that $\left(f_{j}\left(\rho^{\prime}\right)\right)_{j \in J_{\ell}^{\prime}} \in D_{\ell}$ :

In this case all functions $f_{j}(z), j \in J_{\ell}^{\prime}$, with $\rho_{j}=\rho^{\prime}$ have to be (again) of type (10). Consequently $\delta(z)$ behaves like

$$
c_{0}-c_{1}\left(1-z / \rho^{\prime}\right)^{2^{-\tilde{k}}}+\ldots,
$$

where $c_{0}>0$ and $c_{1}>0$ and $\tilde{k}$ is the largest appearing $k_{j}$ (among those functions $f_{j}(z)$ with $\left.\rho_{j}=\rho^{\prime}\right)$. Hence, $1 / \delta(z)$ is of type (10).

Note that there are no other subcases. This follows from the fact that $\rho\left(\mathbf{u}_{\ell}\right) \rightarrow 0$ if $\mathbf{u}_{\ell}$ approaches the boundary of $D_{\ell}$. This means that if we trace the function $z \rightarrow \delta(z)$ for $z>0$ then we either meet a singularity of $\delta(z)$ or we pass a zero of $\delta(z)$ before $\left.\left(f_{j}(z)\right)_{j \in J_{\ell}^{\prime}}\right)$ leaves $D_{\ell}$.

Finally, we have to discuss the numerator (as in the above case). Note that there might occur $u_{j}$ with $j \notin J_{\ell}^{\prime}$, so that more functions $f_{j}(z)$ than in the denominator are involved. Nevertheless in all possible subcases we can combine the expansions of the numerator and denominator and obtain for $f(z)$ either type (10) or type (11).

(3) Finally, let us assume that $\mathbf{f}_{\ell}\left(z, \mathbf{u}_{\ell}\right)$ comes from a non-affine system and, thus, has a square-root singularity. Again, since all functions in $\mathbf{f}_{\ell}\left(z, \mathbf{u}_{\ell}\right)$ have the same form we just consider one of these functions and denote it by $f\left(z, \mathbf{u}_{\ell}\right)$ :

$$
f\left(z, \mathbf{u}_{\ell}\right)=g\left(z, \mathbf{u}_{\ell}\right)-h\left(z, \mathbf{u}_{\ell}\right) \sqrt{1-\frac{z}{\rho\left(\mathbf{u}_{\ell}\right)}} .
$$

In this case $\rho\left(\mathbf{u}_{\ell}\right)$ depends on all components of $\mathbf{u}_{\ell}$ which makes the analysis slightly more easy. As above we will study the behavior of the square-root $\sqrt{\rho\left(\mathbf{u}_{\ell}\right)-z}$ instead of $\sqrt{1-z / \rho\left(\mathbf{u}_{\ell}\right)}$ since the non-zero factor $\sqrt{\rho\left(\mathbf{u}_{\ell}\right)}$ can be put to $h\left(z, \mathbf{u}_{\ell}\right)$.

Let $\rho^{\prime}$ denote the smallest radius of convergence of the functions $f_{j}(z)$ that correspond to $\mathbf{u}_{\ell}$. Here we have to consider the following subcases:

(3.1) $\delta\left(\rho^{\prime \prime}\right)=0$ for some $\rho^{\prime \prime}<\rho^{\prime}$ such that $\left(f_{j}\left(\rho^{\prime \prime}\right)\right) \in D_{\ell}$ :

This means that $\rho\left(\left(f_{j}(z)\right)-z\right.$ has a simple zero. Thus, we can represent this 
function as

$$
\rho\left(\left(f_{j}(z)\right)-z=\left(\rho^{\prime \prime}-z\right) H(z)\right.
$$

where $H(z)$ is non-zero and analytic at $\rho^{\prime \prime}$. Consequently

$$
\sqrt{\rho\left(\left(f_{j}(z)\right)-z\right.}=\sqrt{\rho^{\prime \prime}-z} \sqrt{H(z)}
$$

and we observe that $f(z)$ has a (simple) square-root singularity.

(3.2) We have $\delta\left(\rho^{\prime}\right)=0$ such that $\left(f_{j}\left(\rho^{\prime}\right)\right) \in D_{\ell}$ :

In this case all functions $f_{j}(z)$ with $\rho_{j}=\rho^{\prime}$ have to be of type (10). Hence the square-root of $\delta(z)$ behaves as

$$
\sqrt{c\left(1-z / \rho^{\prime}\right)^{2^{-\tilde{k}}}+\ldots}=\sqrt{c}\left(1-z / \rho^{\prime}\right)^{2^{-\tilde{k}-1}}+\ldots,
$$

where $\tilde{k}$ equals the largest appearing $k_{j}$ plus 1 . Thus, $f(z)$ is of type $(10)$.

(3.3) We have $\delta\left(\rho^{\prime}\right)>0$ such that $\left(f_{j}\left(\rho^{\prime}\right)\right)_{j \in J_{\ell}^{\prime}} \in D_{\ell}$ :

In this case all functions $f_{j}(z)$, with $\rho_{j}=\rho^{\prime}$ have to be (again) of type (10).

Consequently the square-root of $\delta(z)$ behaves like

$$
\sqrt{c_{0}-c_{1}\left(1-z / \rho^{\prime}\right)^{2^{-\tilde{k}}}+\ldots}=\sqrt{c_{0}}\left(1-\frac{c_{1}}{2 c_{0}}\left(1-z / \rho^{\prime}\right)^{2^{-\tilde{k}}}+\ldots\right),
$$

where $c_{0}>0$ and $c_{1}>0$ and $\tilde{k}$ is the largest appearing $k_{j}$ (among those functions $f_{j}(z)$ with $\left.\rho_{j}=\rho^{\prime}\right)$. Hence, $f(z)$ is of type (10).

This completes the induction proof of Theorem 4 .

\section{Periodicities}

When we are interested in the asymptotic properties of the coefficients of $\mathbb{R}_{+}{ }^{-}$ algebraic equations, we need the structure of all singularities $z$ on the radius of convergence $|z|=\rho$. When they are several such singularities, some periodic behaviors can appear and justify the following definition:

Definition 6 (strong (a)periodicity). We will call a function $f(z)$ that is solution of a positive system of algebraic equations strongly aperiodic if $z=\rho$ is the only singularity on the circle $|z|=\rho$.

Similarly we call such a function $f(z)$ strongly periodic with period $p>1$ if $f(z)$ is not strongly aperiodic but can be represented as $f(z)=\sum_{j=0}^{p} z^{j} f_{j}\left(z^{p}\right)$ such that all functions $f_{j}(z)$ are either polynomials or strongly aperiodic functions and at least one of these functions is strongly aperiodic.

The main purpose of this section is to prove the following property.

Theorem 5. Every function $f(z)$ that is solution of an analytically well defined positive polynomial system of equations (see Definition 5 of Section 5) is either strongly aperiodic or strongly periodic (with some period $p>1$ ).

In particular this implies the following asymptotic relations for the coefficients of solutions of a positive polynomial system.

Theorem 6. Suppose that $f(z)$ is a solution of an analytically well defined positive polynomial system of equations (see Definition 5 of Section 5). Then there exists an integer $p \geq 1$ such that for all $j=0,1, \ldots, p-1$ we either have $f_{n}=0$ for almost all $n \geq n_{0, j}$ with $n \equiv j \bmod p$ or

$$
f_{n} \sim C_{j} n^{\alpha_{j}} \rho_{j}^{-n} \quad(n \rightarrow \infty, n \equiv j \bmod p),
$$


where $C_{j}>0, \rho_{j}>0$, and $\alpha_{j}$ is either of the form $\alpha_{j}=-2^{-k_{j}}-1$ for some integer $k_{j} \geq 1$ or of the form $\alpha_{j}=m_{j} / 2^{k_{j}}-1$ for some integers $k_{j} \geq 0$ and $m_{j} \geq 1$.

Proof. If $f(z)$ is strongly aperiodic then the radius of convergence $\rho$ is the only singularity on the circle $|z|=\rho$ and the kind of possible singularities is given by Theorem 4. Furthermore since $f(z)$ is an algebraic function it can be analytically continued to a region of the form $\{z \in \mathbb{C}:|z|<\rho+\eta\} \backslash[\rho, \infty)$ for some $\eta>0$. Consequently we can apply the transfer principle of Flajolet and Odlyzko [62] and obtain the proposed asymptotic expansion for the coefficients.

In the periodic case we just apply this for $f_{j}(z), 0 \leq j<p$.

The proof of Theorem 5 runs along similar lines as the proof of Theorem 4, that is, we partition the dependency graph into strongly connected components and solve the system step by step. The core of the proof is to check in every step that each solution is strongly aperiodic or strongly periodic.

For this purpose we will have to split the solution functions into several parts.

Lemma 5. Suppose that $\mathbf{y}=\mathbf{P}(z, \mathbf{y})$ is an analytically well defined positive polynomial system of equations and $\mathbf{y}=\mathbf{f}(z)=\left(f_{1}(z), \ldots, f_{d}(z)\right)$ is the solution. Then for every $p \geq 1$ we can represent $f_{k}(z), 1 \leq k \leq d$, as $f_{k}(z)=\sum_{j=0}^{p-1} z^{j} f_{k, j}\left(z^{p}\right)$ and the functions $f_{k, j}(z)-f_{k, j}(0), 1 \leq k \leq d, 0 \leq j<p$, that are no polynomials are again solutions of an analytically well defined positive polynomial system of equations $\tilde{\mathbf{y}}=\tilde{\mathbf{P}}(z, \tilde{\mathbf{y}})$

Proof. Let $\mathbf{y}=\mathbf{P}(z, \mathbf{y})$ be an analytically well defined positive system of polynomial equations that has $y_{1}=f(z)$ as one of its solutions. By substituting $\mathbf{y}=\sum_{j=0}^{p-1} z^{j} \mathbf{y}_{j}$, and expanding the polynomials of $\mathbf{P}(z, \mathbf{y})$ it follows that we can represent it as

$$
\mathbf{P}(z, \mathbf{y})=\sum_{j=0}^{p-1} z^{j} \mathbf{P}_{j}\left(z^{p}, \mathbf{y}_{0}, \ldots, \mathbf{y}_{p-1}\right) .
$$

Hence, if we consider the $p \times d$-dimensional system

$$
\mathbf{y}_{j}=\mathbf{P}_{j}\left(z, \mathbf{y}_{0}, \ldots, \mathbf{y}_{p-1}\right), \quad 1 \leq j<p
$$

then with $\tilde{\mathbf{y}}=\left(\mathbf{y}_{0}, \ldots, \mathbf{y}_{p-1}\right)$ and $\tilde{\mathbf{P}}=\left(\mathbf{P}_{0}, \ldots, \mathbf{P}_{p-1}\right)$ we obtain a proper positive polynomial system $\tilde{\mathbf{y}}=\tilde{\mathbf{P}}(z, \tilde{\mathbf{y}})$, where the functions $f_{k, j}(z)$ (that are defined by $\left.f_{k}(z)=\sum_{j=0}^{p-1} z^{j} f_{k, j}\left(z^{p}\right)\right)$ are solutions. Of course, if $f_{k, j}(0)>0$ we can shift the system to have solutions $\left.f_{k, j}(z)-f_{k, j}(0)\right)$ and we can remove polynomial solutions from the system.

The final step is to show that the spectral radius of the Jacobian $\tilde{\mathbf{P}}_{\tilde{\mathbf{y}}}$ (for $z=0$ and $\left.\left.y_{k, j}=f_{k, j}(0)\right)\right)$ is again smaller than 1 . Actually it is an easy exercise to show that the spectral radii are the same. More precisely if $\lambda$ is a positive eigenvalue of $\tilde{\mathbf{P}}_{\tilde{\mathbf{y}}}$ with a positive eigenvector then it is a positive eigenvalue of $\mathbf{P}_{\mathbf{y}}$ (with a corresponding positive eigenvector), too. We illustrate the idea of the proof in a slightly simplified situation. Suppose that we have the system $\mathbf{y}=\mathbf{P}(z, \mathbf{y})$ and we write $\mathbf{y}=\mathbf{y}_{1}+\mathbf{y}_{2}$ and $\mathbf{P}\left(z, \mathbf{y}_{1}+\mathbf{y}_{2}\right)=\mathbf{P}_{1}\left(z, \mathbf{y}_{1}, \mathbf{y}_{2}\right)+\mathbf{P}_{1}\left(z, \mathbf{y}_{1}, \mathbf{y}_{2}\right)$ and consider the extended system $\mathbf{y}_{1}=\mathbf{P}_{1}\left(z, \mathbf{y}_{1}, \mathbf{y}_{2}\right), \mathbf{y}_{2}=\mathbf{P}_{2}\left(z, \mathbf{y}_{1}, \mathbf{y}_{2}\right)$. Let $\lambda>0$ be an eigenvalue of

$$
\left(\begin{array}{ll}
\mathbf{P}_{1, \mathbf{y}_{1}} & \mathbf{P}_{1, \mathbf{y}_{2}} \\
\mathbf{P}_{2, \mathbf{y}_{1}} & \mathbf{P}_{2, \mathbf{y}_{2}}
\end{array}\right)
$$


with a positive eigenvector $\mathbf{x}=\left(\mathbf{x}_{1}, \mathbf{x}_{2}\right)$, that is,

$$
\left(\begin{array}{ll}
\mathbf{P}_{1, \mathbf{y}_{1}} & \mathbf{P}_{1, \mathbf{y}_{2}} \\
\mathbf{P}_{2, \mathbf{y}_{1}} & \mathbf{P}_{2, \mathbf{y}_{2}}
\end{array}\right)\left(\begin{array}{c}
\mathbf{x}_{1} \\
\mathbf{x}_{2}
\end{array}\right)=\lambda\left(\begin{array}{l}
\mathbf{x}_{1} \\
\mathbf{x}_{2}
\end{array}\right) .
$$

By multiplying from the left with $(\mathbf{I}, \mathbf{I})$ and by observing that $\mathbf{P}_{\mathbf{y}}=\mathbf{P}_{\mathbf{y}_{1}}=\mathbf{P}_{1, \mathbf{y}_{1}}+$ $\mathbf{P}_{2, \mathbf{y}_{1}}=\mathbf{P}_{\mathbf{y}_{2}}=\mathbf{P}_{1, \mathbf{y}_{2}}+\mathbf{P}_{2, \mathbf{y}_{2}}$ we, thus, obtain

$$
\mathbf{P}_{\mathbf{y}}\left(\mathbf{x}_{1}+\mathbf{x}_{2}\right)=\lambda\left(\mathbf{x}_{1}+\mathbf{x}_{2}\right) \text {. }
$$

It is clear now how we can adapt this example to the original situations.

Lemma 6. Suppose that $f(z)=\sum_{n \geq 0} a_{n} z^{n}$ is a strongly aperiodic function with non-negative coefficients $a_{n}$ and radius of convergence $\rho$. Then for every $p \geq 1$ we can represent $f(z)$ as

$$
f(z)=\sum_{j=0}^{p-1} z^{j} f_{j}\left(z^{p}\right),
$$

where the functions $f_{j}(z)=\sum_{n \geq 0} a_{j+p n} z^{n}, 0 \leq j<m$, are strongly aperiodic and have the same kind of dominating singularity.

Proof. By Lemma 5 we already know that $f_{j}(z)$ is solution of an analytically well defined positive polynomial system of equations. Furthermore $f_{j}(z)$ can be represented as

$$
f_{j}(z)=\frac{1}{p} \sum_{\ell=0}^{p-1} e^{-2 \pi i j \ell / p} f\left(z^{1 / p} e^{2 \pi i \ell / p}\right) .
$$

Since $f(z)$ is strongly aperiodic the radius of convergence $\rho$ is the unique singularity on the circle of convergence $|z|=\rho$. Hence, $\rho^{1 / p}$ is the radius of convergence of $f_{j}(z)$ and (again) the only singularity of $f_{j}(z)$ on the circle of convergence $|z|=\rho^{1 / p}$. Since the coefficients $\left(a_{j+p n}\right)_{n \geq 0}$ have the same kind of asymptotic expansion as $\left(a_{n}\right)_{n \geq 0}$, it also follows that $f_{j}(z)$ ( for $z \sim \rho^{1 / p}$ ) and $f(z)$ (for $z \sim \rho$ ) have the same kind of singularity.

We start our considerations concerning the proof of Theorem 5 with a strongly connected affine system. In order to make the statements (and proofs) simpler we assume that we have already reduced the system to a single equation of the form $y=a(z) y+b(z)$, where $a(z)$ and $b(z)$ are rational functions with non-negative coefficients that are regular for $|z|<\rho+\varepsilon$ for some $\varepsilon>0$, where $\rho>0$ is given by $a(\rho)=1$ and is the radius of convergence of $f(z)=b(z) /(1-a(z))$.

Lemma 7. Suppose that $a(z)$ and $b(z)$ are non-zero rational functions with nonnegative coefficients that are regular for $|z| \leq \rho$, where $\rho>0$ is given by $a(\rho)=1$. Furthermore we assume that $a^{\prime}(z) \neq 0$.

Then $f(z)$ is strongly aperiodic or strongly periodic (with period p for some integer $p>1$ ) such that all singularities on the circle of convergence are poles of order 1.

We note that this lemma can be generalized to functions $f(z)$ that are solutions of not necessarily strongly connected affine systems, see [99, Theorem 10.1]. The only difference is that the order of poles might be larger than 1 in the not strongly connected case, however, the order of $\rho$ is the maximum appearing order. 
Proof. Let $a(z)=\sum_{n \geq 0} a_{n} z^{n}$. Since $a_{n} \geq 0$ and $a(z) \neq 0$ it is clear that there exists a unique $\rho>0$ with $a(\rho)=1$ which is (by assumption) the radius of convergence and also a polar singularity of $f(z)$. Now suppose that $z=\rho \zeta$ is also a singularity of $f(z)$, where $|\zeta|=1$. Then we certainly have $a(\rho \zeta)=1$. On the other hand we have

$$
|a(\rho \zeta)|=\left|\sum_{n \geq 0} a_{n} \rho^{n} \zeta^{n}\right| \leq \sum_{n \geq 0} a_{n} \rho^{n}=1 .
$$

which implies that all inequalities have to be equalities. In particular we have $a_{p} \rho^{p} \zeta^{p}=a_{p} \rho^{p}$ for some $p>0$ for which $a_{p}>0$. Consequently $\zeta^{m}=1$. Thus we are certainly in the strongly aperiodic or strongly periodic case.

The example

$$
f(z)=\frac{1}{1-z}+\frac{1}{1-z^{2}}=\frac{2+z}{1-z^{2}}
$$

shows that even a single equation of the form $y=z^{2} y+2+z$ can lead to a (strongly) periodic case with period $p=2>1$, where the behavior in both residue classes is different and non-zero. However, if we use the method of Lemma 5 we can reduce this equation to a system with only strongly aperiodic solutions. If we set $y=y_{0}+z y_{1}$ then we have

$$
z^{2} y+2+z=z^{2}\left(y_{0}+z y_{1}\right)+2+z=\left(2+z^{2} y_{0}\right)+z\left(1+z^{2} y_{1}\right)
$$

Hence, if we consider the system $\left\{y_{0}=2+z y_{0}, y_{1}=1+z y_{1}\right\}$, then we have as solutions $f_{0}(z)=2 /(1-z)$ and $f_{1}(z)=1 /(1-z)$ which are strongly aperiodic and give back the original solutions $f(z)$ as

$$
f(z)=f_{0}\left(z^{2}\right)+z f_{1}\left(z^{2}\right)
$$

It is interesting to observe that in non-affine and strongly connected systems there is only one residue class modulo $p$, where the coefficients are non-zero. This is proved in the next lemma. As in the affine case we assume that we have already reduced the system of equations to a single equation; as in the affine case the right hand side of the equation is not anymore a polynomial, but an algebraic function. Actually the reduction procedure (compare also with the proof of Lemma 4) leads to an equation that satisfies the following regularity conditions.

Lemma 8. Suppose that $P(z, y)=\sum_{k, \ell} a_{k \ell} z^{k} y^{\ell}$ is an algebraic function with nonnegative coefficients such that $a_{k \ell}>0$ for some $\ell \geq 2$ and $a_{01}<1$. Furthermore let $\rho>0$ denote the radius of convergence of the solution $f(z)$ of the equation $y=P(z, y)$ and suppose that there exist $\varepsilon>0$ such that $P(z, y)$ is regular for $|z|<\rho+\varepsilon$ and $|y|<f(\rho)+\varepsilon$.

Let $p$ be the largest positive integer for which there exists an integer $r \geq 0$ such that $P(z, 0)$ can be represented as $P(z, 0)=z^{r} q\left(z^{p}\right)$ for a proper function $q(z)$ with non-negative coefficients and that $p$ divides $k+r(\ell-1)$ for all $a_{k \ell}>0$ with $\ell>0$. If $p=1$ then $f(z)$ is strongly aperiodic and if $p>1$ then $f(z)$ is strongly periodic with period $p$ and can be represented as $f(z)=z^{r} \tilde{f}\left(z^{p}\right)$, where $\tilde{f}(z)$ is strongly aperiodic.

Proof. Let $\rho>0$ be the radius of convergence of $f(z)$ and $\eta=f(\eta)>0$. Then we have $P(\rho, \eta)=\eta$ and $P_{y}(\rho, \eta)=1$. If $\left|z^{\prime}\right|=\rho$ then we have $\left|f\left(z^{\prime}\right)\right| \leq f\left(\left|z^{\prime}\right|\right)=\eta$ and consequently $\left|P_{y}\left(z^{\prime}, f\left(z^{\prime}\right)\right)\right| \leq P_{y}\left(\left|z^{\prime}\right|,\left|f\left(z^{\prime}\right)\right|\right) \leq P_{y}\left(\left|z^{\prime}\right|, f\left(\left|z^{\prime}\right|\right)\right)=1$. Hence, if $z^{\prime}$ is a singularity, that is, we certainly have $P_{y}\left(z^{\prime}, f\left(z^{\prime}\right)\right)=1$, then all these inequalities have to be equalities. From $\left|f\left(z^{\prime}\right)\right|=f\left(\left|z^{\prime}\right|\right)$ it follows (similarly to the proof of 
Lemma 7) that $f(z)$ can be written as $f(z)=z^{r} \tilde{f}\left(z^{p}\right)$ (for some integers $r \geq 0$ and $p \geq 1$ ) and $z^{\prime}$ is of the form $z^{\prime}=\rho e^{2 \pi j / p}$ (for some integer $j$ that is coprime to $m$ ). Consequently from $P_{y}\left(z^{\prime}, f\left(z^{\prime}\right)\right)=1$ and $f\left(z^{\prime}\right)=\eta e^{2 \pi i j r / p}$ it follows that $p$ divides $k+r(\ell-1)$ for all pairs $(k, \ell)$ for which $a_{k \ell}>0$ and $\ell>0$. Finally, we also have $f\left(z^{\prime}\right)=P\left(z^{\prime}, f\left(z^{\prime}\right)\right)$ which implies that $P(z, 0)$ can be represented as $P(z, 0)=z^{r} q\left(z^{p}\right)$ for a polynomial $q$.

Conversely we can search for the largest positive integer $p$ for which there exists an integer $r \geq 0$ such that $P(z, 0)$ can be represented as $P(z, 0)=z^{r} q\left(z^{p}\right)$ and that $p$ divides $k+r(\ell-1)$ for all pairs $(k, \ell)$ with $a_{k \ell}>0$ and $\ell>0$. It is clear that the power series of $f(z)$ is divisible by $z^{r}$. Furthermore we can represent $P$ as

$$
P(z, y)=z^{r} q\left(z^{p}\right)+z^{r} \sum_{k \geq 0, \ell \geq 1} a_{k, \ell} z^{k+r(\ell-1)}\left(y / z^{r}\right)^{\ell}=z^{r} Q\left(z^{p}, y / z^{r}\right)
$$

for some proper function $Q$. Hence $\bar{y}=y / z^{r}$ solves the equation $\bar{y}=Q\left(z^{p}, \bar{y}\right)$ and can be represented as $\bar{f}(z)=\tilde{f}\left(z^{p}\right)$. This implies that $f(z)=z^{r} \tilde{f}\left(z^{p}\right)$. Finally, since $p$ was chosen to be the largest integer satisfying the above mentioned properties it follows that $\tilde{f}(z)$ is strongly aperiodic. Otherwise we could iterate the procedure and obtain a contradiction.

In order to complete the proof of Theorem 5, we now follow the proof of Theorem 4 and observe step by step that all functions are strongly aperiodic or strongly periodic. For this purpose we will frequently use the set $\mathcal{F}$ of algebraic functions $f$ for which there exists $p \geq 1$ and (algebraic) functions $f_{j}, 0 \leq j<p$, with $f(z)=\sum_{j=0}^{p} z^{j} f_{j}\left(z^{p}\right)$ and the functions $f_{j}, 0 \leq j<p$, have positive radii of convergence which are the only singularities on the circles of convergence and all dominating singularities are of the types described in Theorem 4. This set of functions has the following property.

Lemma 9. The set $\mathcal{F}$ is closed under taking sums and products.

Furthermore suppose that $f \in \mathcal{F}$ has only one singularity $\rho$ on the circle of convergence and $f(\rho)$ is finite. Furthermore let $c(z, u)$ be a power series $c(z, u)=$ $\sum_{n, k} a_{n, k} z^{n} u^{k}$ with non-negative coefficients $a_{n, k}$ that is analytic at $(\rho, f(\rho))$ and there is some $n$ and some $k>0$ with $a_{n, k}>0$. Then the function $g(z)=c(z, f(z))$ is in $\mathcal{F}$, too, and has again the property that $\rho$ is the only one singularity on the circle of convergence.

Proof. Suppose that $f$ and $g$ are in $\mathcal{F}$ and have radii of convergence $\rho_{1}$ and $\rho_{2}$ and periods $p_{1}$ and $p_{2}$. Of course, we only have to consider the case $\rho_{1}=\rho_{2}$.

If $p_{1}=p_{2}=1$ it is immediate to see that $f+g$ and $f \cdot g$ are in $\mathcal{F}$.

If $p_{1}>1$ or $p_{2}>1$ then let $p$ denote the smallest common multiple of $p_{1}$ and $p_{2}$. With the help of Lemma 6 it follows that we can represent $f$ and $g$ as

$$
f(z)=\sum_{j=0}^{p-1} z^{j} f_{j}\left(z^{p}\right) \quad \text { and } \quad g(z)=\sum_{j=0}^{p-1} z^{j} g_{j}\left(z^{p}\right)
$$

where $f_{j}$ and $g_{j}$ have the property that there is only one singularity on the circle of convergence. Hence, it follows similarly to the case $p_{1}=p_{2}=1$ that $f+g$ and $f \cdot g$ are in $\mathcal{F}$.

Finally, in order to handle the function $g(z)=c(z, f(z))$ we just have to observe (by a local expansion) that $g(z)$ has the same kind of singularity as $f(z)$ (at $z=\rho$ ) and that there are no other singularities for $|z| \leq \rho$ other than $\rho$. 
In order to prove Theorem 5 we show now inductively that all appearing solution functions of an analytically well defined systems are contained in $\mathcal{F}$.

Suppose that we are considering a strongly connected component $C_{\ell}$ and the corresponding system $\mathbf{y}_{\ell}=\mathbf{P}_{\ell}\left(z, \mathbf{y}_{\ell}, \mathbf{u}_{\ell}\right)$, where $\mathbf{u}_{\ell}$ denotes the input vector that corresponds to those components that have been already solved before (and for which we can assume by induction that they are in $\mathcal{F}$ ).

As in the proof of Theorem 4 we distinguish between three cases:

(1) First let us assume that $\mathbf{f}_{\ell}\left(z, \mathbf{u}_{\ell}\right)$ comes from an affine system of the form $\mathbf{y}_{\ell}=\mathbf{A}_{\ell}(z) \mathbf{y}_{\ell}+\mathbf{B}_{\ell}\left(z, \mathbf{u}_{\ell}\right)$, that is, the matrix $\mathbf{I}-\mathbf{A}_{\ell}(z)$ does not depend on $\mathbf{u}$ or equivalently

$$
f\left(z, \mathbf{u}_{\ell}\right)=\frac{c\left(z, \mathbf{u}_{\ell}\right)}{1-z / \rho^{\prime}},
$$

where $c\left(z, \mathbf{u}_{\ell}\right)$ is a polynomial in $\mathbf{u}_{\ell}$ with non-negative coefficients. Note that the coefficient functions $c_{j}(z) /\left(1-z / \rho^{\prime}\right)$ are in $\mathcal{F}$.

When we substitute $\mathbf{u}_{\ell}$ by the functions $f_{j}(z)$ that correspond to $\mathbf{u}_{j}$ (and, thus, are in $\mathcal{F}$ ) then it follows from Lemma 9 that the resulting function is in $\mathcal{F}$.

(2) Second let us (again) assume that $\mathbf{f}_{\ell}\left(z, \mathbf{u}_{\ell}\right)$ comes from an affine system $\mathbf{y}_{\ell}=\mathbf{A}_{\ell}\left(z, \mathbf{u}_{\ell}\right) \mathbf{y}_{\ell}+\mathbf{B}_{\ell}\left(z, \mathbf{u}_{\ell}\right)$, where $\mathbf{A}_{\ell}\left(z, \mathbf{u}_{\ell}\right)$ depends on some of the $u_{j}$. As above let $J_{\ell}^{\prime}$ denote the set of indices of functions $u_{j}$ on which $\mathbf{A}_{\ell}\left(z, \mathbf{u}_{\ell}\right)$ really depends. Of course, if we represent $f\left(z, \mathbf{u}_{\ell}\right)$ as

$$
f\left(z, \mathbf{u}_{\ell}\right)=\frac{c\left(z, \mathbf{u}_{\ell}\right)}{1-z / \rho\left(\mathbf{u}_{\ell}\right)}
$$

then $J_{\ell}^{\prime}$ is precisely the set of indices of functions $u_{j}$ on which the function $\rho\left(\mathbf{u}_{\ell}\right)$ depends. Note that $c\left(z, \mathbf{u}_{\ell}\right)$ is a polynomial in the $u_{j}$ with $j \notin J_{\ell}^{\prime}$. (In what follows we will denote the coefficients of this polynomial by $c_{r}\left(z,\left(u_{j}\right)_{j \in J_{\ell}^{\prime}}\right)$. We note that for all $r$ the function $c_{r}\left(z,\left(u_{j}\right)_{j \in J_{\ell}^{\prime}}\right) /(1-$ $\left.z / \rho\left(u_{j}\right)_{j \in J_{\ell}^{\prime}}\right)$ is a power series with non-negative coefficients in $z$ and $\left(u_{j}\right)_{j \in J_{\ell}^{\prime}}$.)

Let $\rho^{\prime}$ denote the smallest radius of convergence of the functions $f_{j}(z)$, $j \in J_{\ell}^{\prime}$. Then we consider the difference $\delta(z)=\rho\left(\left(f_{j}(z)\right)_{j \in J_{\ell}^{\prime}}\right)-z$. We have to consider the following cases:

(2.1) $\delta\left(\rho^{\prime \prime}\right)=0$ for some $\rho^{\prime \prime}<\rho^{\prime}$ such that $\left(f_{j}\left(\rho^{\prime \prime}\right)\right)_{j \in J_{\ell}^{\prime}} \in D_{\ell}$ :

Here we have a polar singularity and with the help of Lemma 7 we deduce that $c_{r}\left(z,\left(f_{j}(z)\right)_{j \in J_{\ell}^{\prime}}\right) /\left(1-z / \rho\left(\left(f_{j}(z)\right)_{j \in J_{\ell}^{\prime}}\right)\right)$ is in $\mathcal{F}$. Hence it follows (again) from Lemma 9 that the resulting function is in $\mathcal{F}$.

(2.2) We have $\delta\left(\rho^{\prime}\right)=0$ such that $\left(f_{j}\left(\rho^{\prime}\right)\right)_{j \in J_{\ell}^{\prime}} \in D_{\ell}$ :

In this case we first replace the system $\mathbf{y}_{\ell}=\mathbf{A}_{\ell}\left(z, \mathbf{u}_{\ell}\right) \mathbf{y}+\mathbf{B}_{\ell}\left(z, \mathbf{u}_{\ell}\right)$ by another one. By assumption we know that all functions $f_{j}(z)$ are strongly aperiodic (with periods $p_{j}$ ). We define $p$ as the least common multiple of the periods $p_{j}$ and by Lemma 6 we can represent them all as $f_{j}(z)=\sum_{i=0}^{p-1} z^{i} f_{j, i}\left(z^{p}\right)$ with strongly aperiodic functions $f_{j, i}(z)$. Formally this means that we replace the parameters $u_{j}$ by $\sum_{i=0}^{p-1} z^{i} u_{j, i}$. Furthermore by following the proof method of Lemma 5 we split the (original $d$-dimensional) system into a $p \times d$ dimensional system, where the solutions $f_{i}(z)$ correspond to the original one by $f(z)=\sum_{i=0}^{p-1} z^{i} f_{i}\left(z^{p}\right)$. Let us denote this new system by 
$\tilde{\mathbf{y}}_{\ell}=\tilde{\mathbf{A}}_{\ell}\left(z, \tilde{\mathbf{u}}_{\ell}\right) \tilde{\mathbf{y}}_{\ell}+\tilde{\mathbf{B}}_{\ell}\left(z, \tilde{\mathbf{u}}_{\ell}\right)$. It is easy to check that this new system is either strongly connected or the corresponding dependency graph decomposes into several strongly connected components without any link between these components. For the sake of simplicity we assume that we have only one component (in the other case we have to deal with each component separately, however, this does not produce any difficulty).

The advantage of this construction is that all functions $f_{j, i}(z)$ (that have to be substituted for $u_{j, i}$ ) are strongly aperiodic which implies that $\left|f_{j, i}(z)\right|<f_{j, i}(|z|)$ if $z$ is not a positive real number.

It might occur that the new system (or one of the new systems) falls into the cases (2.1) or (2.3). In these cases we proceed as explained there. Thus we can assume that we are again in case (2.2). We note that the function $\tilde{\rho}\left(\tilde{\mathbf{u}}_{\ell}\right)$ is determined by the property that the positive matrix $\tilde{\mathbf{A}}_{\ell}\left(z, \tilde{\mathbf{u}}_{\ell}\right)$ has spectral radius 1 . Since this matrix is irreducible it follows that the spectral radius is strictly smaller than 1 if at least one of the entries decreases in modulus. Hence if we substitute the $u_{j, i}$ by $f_{j, i}(z)$ there is certainly no singularity if $|z|=\tilde{\rho}^{\prime}$ but $z \neq \tilde{\rho}^{\prime}$. Consequently the only singularity of the resulting function is on the circle of convergence $z=\tilde{\rho}^{\prime}$ and we know already from the proof of Theorem 4 which kind of singularity will appear.

Summing up it follows that the solution of the expanded system is strongly aperiodic and, thus, the solution of the original system is either strongly aperiodic or strongly periodic.

(2.3) We have $\delta\left(\rho^{\prime}\right)>0$ such that $\left(f_{j}\left(\rho^{\prime}\right)\right)_{j \in J_{\ell}^{\prime}} \in D_{\ell}$ :

We first apply the same transformation of the system as in case (2.2) and are led to one (or several) strongly dependent new system. Of course some of them might be of types (2.1) or (2.2). But then we just apply the procedure there; however, it is important to note that it is not necessary to apply the transformation again, since we have already reached the goal that all functions $f_{j, i}(z)$ are strongly aperiodic.

In order to simplify the notation we stick with the original notation of the system $\mathbf{y}_{\ell}=\mathbf{A}_{\ell}\left(z, \mathbf{u}_{\ell}\right) \mathbf{y}_{\ell}+\mathbf{B}_{\ell}\left(z, \mathbf{u}_{\ell}\right)$.

In this case we can represent the function $f(z)$ that results after substitution as a polynomial in $f_{j}(z)$ with $j \notin J^{\prime}$ with coefficient functions that are analytic in $z$ and $\left(f_{j}(z)\right)_{j \in J^{\prime}}$ at $z=\rho^{\prime}$ and $\left(f_{j}\left(\rho^{\prime}\right)\right)_{j \in J^{\prime}}$. Of course all coefficients in the power series expansions are non-negative. By extending the last part of Lemma 9 to several (strongly aperiodic) functions it follows that the coefficients functions are in $\mathcal{F}$ which implies (by another application of Lemma 9) that the resulting function is in $\mathcal{F}$.

(3) Finally, let us assume that $\mathbf{f}_{\ell}\left(z, \mathbf{u}_{\ell}\right)$ comes from a non-affine system and, thus, has a square-root singularity of the form (16). In this case $\rho\left(\mathbf{u}_{\ell}\right)$ depends on all components of $\mathbf{u}_{\ell}$. As above we set $\delta(z)=\rho\left(\mathbf{u}_{\ell}\right)-z$ and let $\rho^{\prime}$ denote the smallest radius of convergence of the functions $f_{j}(z)$ that correspond to $\mathbf{u}_{\ell}$.

(3.1) $\delta\left(\rho^{\prime \prime}\right)=0$ for some $\rho^{\prime \prime}<\rho^{\prime}$ such that $\left(f_{j}\left(\rho^{\prime \prime}\right)\right) \in D_{\ell}$ :

Here we are precisely in the situation of Lemma 8 . From the very 
beginning on we can substitute $u_{j}$ by the functions $f_{j}(z)$ and thus obtain either a strongly aperiodic function or a strongly periodic function, where only one residue class modulo $m$ appears.

(3.2) We have $\delta\left(\rho^{\prime}\right)=0$ such that $\left(f_{j}\left(\rho^{\prime}\right)\right) \in D_{\ell}$ :

As in case (2.2) we first replace the original system $\mathbf{y}_{\ell}=\mathbf{P}_{\ell}\left(z, \mathbf{y}_{\ell}, \mathbf{u}_{\ell}\right)$ by a new one $\tilde{\mathbf{y}}=\tilde{\mathbf{P}}_{\ell}\left(z, \tilde{\mathbf{y}}_{\ell}, \tilde{\mathbf{u}}_{\ell}\right)$ which might decompose into several strongly connected but non-affine systems. As above we assume that it is just one system and that it is again of type (3.2). Here the function $\tilde{\rho}\left(\tilde{\mathbf{u}}_{\ell}\right)$ is given by the property that the Jacobian $\tilde{P}_{\tilde{\mathbf{y}}}\left(z, \tilde{\mathbf{y}}_{\ell}, \tilde{\mathbf{u}}_{\ell}\right)$ has spectral radius 1. As in the proof of part (2.2) the spectral radius is smaller than 1 if at least one entry of this matrix decreases in modulus. This again implies that there are no singularities on the cycle of convergence $|z|=\rho^{\prime}$ other than $\rho^{\prime}$. And the kind of singularity that appears for $z=\rho^{\prime}$ is already known from the proof of Theorem 4 .

Summing up we again get that the solution of the expanded system is strongly aperiodic and, thus, the solution of the original system is either strongly aperiodic of strongly periodic.

(3.3) We have $\delta\left(\rho^{\prime}\right)>0$ such that $\left(f_{j}\left(\rho^{\prime}\right)\right)_{j \in J_{\ell}^{\prime}} \in D_{\ell}$ :

We proceed similarly to the case (2.3). We first expand the system as in case (3.2) and assume (without loss of generality) that the new system is again of type (3.3) (and for the sake of simplicity we stick with the original notation).

In this case the spectral radius of $\mathbf{P}_{\ell, \mathbf{y}_{\ell}}\left(z, \mathbf{y}_{\ell}, \mathbf{u}_{\ell}\right)$ is smaller than 1 for $\mathbf{u}_{\ell}=\left(f_{j}\left(\rho^{\prime}\right)\right)_{j \in J_{\ell}^{\prime}}$. Thus it follows that we can invert the system of equations $\mathbf{y}_{\ell}=\mathbf{P}_{\ell}\left(z, \mathbf{y}_{\ell}, \mathbf{u}_{\ell}\right)$ locally to $\mathbf{y}_{\ell}=\mathbf{Q}\left(z, \mathbf{u}_{\ell}\right)$ which implies that the resulting function $f(z)$ is singular at $z=\rho^{\prime}$ (and we know from the proof of Theorem 4 that the kind of singularities of $y_{j}(z)$ are inherited). Furthermore it follows that there are no other singularities on the circle of convergence $|z|=\rho^{\prime}$ other than $\rho^{\prime}$. This follows from the fact that the spectral radius of the Jacobian stays smaller than one and that the functions $f_{j}(z)$ are not singular for $|z|=\rho^{\prime}, z \neq \rho^{\prime}$.

This completes the proof of Theorem 5 .

\section{Possible Radius of Convergence of $\mathbb{Q}_{+}$-Algebraic functions}

In this section we briefly discuss the radius of convergence $\rho$ that can appear in a positive algebraic system with rational coefficients. Of course, $\rho$ has to be a positive algebraic number; however, it is not immediate whether all positive algebraic numbers actually appear. Let us begin with an assertion for more restricted systems of equations:

Conjecture 1 (Radius of $\mathbb{N}$-rational functions). All positive algebraic numbers $\leq 1$ appear as a radius of convergence of solutions of a positive affine system of equations with integer coefficients.

This conjecture (which we do not believe to be true, e.g. it is a challenge to find an $\mathbb{N}$-rat function with $\left.\rho=\left(1+2^{1 / 3}\right) / 3\right)$ implies the following weaker conjecture (which we believe to be true): 
Conjecture 2 (Radius of $\mathbb{N}$-algebraic functions). All positive algebraic numbers $\leq 1$ appear as a radius of convergence of solutions of a positive algebraic system of equations with integer coefficients.

If $F(z)$ is $\mathbb{N}$-algebraic then $F(a z)$ (for any rational $a$ ) is $\mathbb{Q}_{+}$-algebraic, so it is clear that Conjecture 2 implies the following conjecture:

Conjecture 3 (Radius of $\mathbb{Q}_{+}$-algebraic functions). All positive algebraic numbers appear as a radius of convergence of solutions of a positive algebraic system of equations with rational coefficients.

For each of these conjectures, it is also natural to ask what are the properties of the set of corresponding radii of convergence, e.g. to what extent are they closed under sum or product? In what follows we present some properties of the set of these algebraic numbers which led us to the above conjecture.

Theorem 7. The set $R$ of radii of convergence of $\mathbb{Q}_{+}$-algebraic functions has the following properties:

(1) All positive roots of equations of the form $p(z)=1$, where $p(z)$ is a polynomial with non-negative rational coefficients, are in $R$, in particular all rational numbers and all roots of rational numbers.

(2) If $\rho_{1} \in R$ and $\rho_{2}$ is a radius of convergence that appear in positive rational systems, then $\rho_{1} \rho_{2} \in R$.

(3) All positive quadratic irrational numbers are in $R$.

Proof. (i) Suppose that $p(z)$ is a polynomial with non-negative rational coefficients and $z_{0}$ a positive solution of the equation $p(z)=1$. Then it is certainly the radius of convergence of $y=f(z)$ that satisfies the equation $y=z+p(z) y$. Since $p(0)<1$ this equation is well posed. By setting $p(z)=z / \rho$ or $p(z)=z^{m} / \alpha$, it follows that rational numbers $\rho$ and roots $\rho=\alpha^{1 / m}$ are in $R$.

(ii) The fact that the Hadamard product of an algebraic function with a rational function is algebraic [72] has a non-commutative version [101]; this implies that the Hadamard product of an $\mathbb{N}$-algebraic function with an $\mathbb{N}$-rational function is $\mathbb{N}$-algebraic. The same holds for $\mathbb{Q}_{+}$instead of $\mathbb{N}$.

We first assume that we are in the aperiodic case. Then the asymptotic expansion for the coefficients $a_{n}$ and $b_{n}$ of the $\mathbb{Q}$-algebraic function and the $\mathbb{Q}$-rational function are of the form $a_{n} \sim A n^{\alpha} \rho_{1}^{-n}$ and $b_{n} \sim B n^{\beta} \rho_{2}^{-n}$ so that we can directly consider the Hadamard product $a_{n} b_{n} \sim A B n^{\alpha+\beta}\left(\rho_{1} \rho_{2}\right)^{-n}$ and observe that the radius of convergence $\rho$ of the Hadamard product is just the product $\rho_{1} \rho_{2}$. Since the Hadamard product is $\mathbb{Q}_{+}$-algebraic it follows that $\rho_{1} \rho_{2} \in R$.

Now suppose that $a(z)$ is $\mathbb{Q}_{+}$-algebraic but not aperiodic. Then we can represent $a(z)$ as $a(z)=\sum_{j=0}^{p-1} z^{j} a_{j}\left(z^{p}\right)$, where the functions $a_{j}(z)$ are $\mathbb{Q}_{+}$-algebraic, too, and there is at least one function, say $a_{j_{0}}\left(z^{p}\right)$, that has the same radius of convergence as $a(z)$. Hence, if we consider the function

$$
\tilde{a}(z)=\left(1+z / \rho+(z / \rho)^{2}+\cdots(z / \rho)^{p-1}\right) a_{j_{0}}\left(z^{p}\right)
$$

then $\tilde{a}(z)$ is again $\mathbb{Q}_{+}$-algebraic and the coefficients $\tilde{a}_{n}$ have an asymptotic expansion of the form $\tilde{a}_{n} \sim \tilde{A} n^{\alpha} \rho_{1}^{-n}$ (for all $n$ and not only in a residue class). A similar procedure works for an aperiodic $\mathbb{Q}_{+}$-rational function and we can proceed as above. 
(iii) Finally we suppose that $\rho=\alpha+\beta \sqrt{m}$ is a positive quadratic irrational number (where $\alpha$ and $\beta$ are rational numbers and $m$ is a square-free positive integer). We have to distinguish several cases.

First suppose that $\alpha<0$ and $\beta>0$. Since $\rho=\alpha+\beta \sqrt{m}>0$ this implies $\alpha^{2}-\beta^{2} m<0$. If we set

$$
p(z)=\frac{2 \alpha}{\alpha^{2}-\beta^{2} m} z+\frac{1}{\beta^{2} m-\alpha^{2}} z^{2},
$$

then $p(z)$ has positive coefficients and we also have $p(\alpha+\beta \sqrt{m})=0$. Consequently $\rho$ is in $R$. Moreover we certainly have $c_{n} \sim d \rho^{n}$ for the coefficients of the solution of $y=z+p(z) y$.

Next suppose that $\alpha>0$ and $\beta<0$. In this case we have $\alpha^{2}-\beta^{2} m>0$ so that $\rho$ is root of the polynomial $z^{2}-2 \alpha z+\left(\alpha^{2}-\beta^{2} m\right)=0$ which cannot be written in an equivalent form $p(z)=1$, where $p(z)$ has non-negative coefficients. Here we consider the (rational) system of equations

$$
y_{1}=z+\left(a z+y_{2}\right) y_{1}, \quad y_{2}=b z+c z y_{2},
$$

where $y_{1}=f_{1}(z)$ has the solution

$$
f_{1}(z)=\frac{z}{1-a z-\frac{b z}{1-c z}}=\frac{z(1-c z)}{1-(a+b+c) z+a c z^{2}} .
$$

Consequently if there are non-negative rational numbers $a, b, c$ with $a+b+c=$ $2 \alpha /\left(\alpha^{2}-\beta^{2} m\right)$ and $a c=1 /\left(\alpha^{2}-\beta^{2} m\right)$ then we are done. For a moment set $a=c=1 / \sqrt{\alpha^{2}-\beta^{2} m}$. Then the trivial inequality $\alpha>\sqrt{\alpha^{2}-\beta^{2} m}$ implies

$$
\frac{2 \alpha}{\alpha^{2}-\beta^{2} m}>\frac{2}{\sqrt{\alpha^{2}-\beta^{2} m}}
$$

Hence, by setting

$$
b=\frac{2 \alpha}{\alpha^{2}-\beta^{2} m}-\frac{2}{\sqrt{\alpha^{2}-\beta^{2} m}}
$$

we obtain $a+b+c=2 \alpha /\left(\alpha^{2}-\beta^{2} m\right)$ and $a c=1 /\left(\alpha^{2}-\beta^{2} m\right)$ with non-negative $a, b, c$. The only problem is that $a, b, c$ are not rational (in general). However, we can choose $a$ to be a proper rational approximation of $1 / \sqrt{\alpha^{2}-\beta^{2} m}$ and then set $c=1 /\left(a\left(\alpha^{2}-\beta^{2} m\right)\right)$ and $b=2 \alpha /\left(\alpha^{2}-\beta^{2} m\right)-a-c$. By continuity we can choose this rational approximation in a way that $a, b, c$ are all positive. Consequently $\rho$ is in $R$. Moreover we again have $c_{n} \sim d \rho^{n}$ for the coefficients of the solution of $f_{1}(z)$.

If $\alpha>0$ and $\beta>0$ then we write $\rho=\alpha+\beta \sqrt{m}$ in the form

$$
\alpha+\beta \sqrt{m}=\frac{1}{\alpha^{2}-\beta^{2} m}(\alpha-\beta \sqrt{m})
$$

if $\alpha^{2}-\beta^{2} m>0$ and in the form

$$
\alpha+\beta \sqrt{m}=\frac{1}{\beta^{2} m-\alpha^{2}}(-\alpha+\beta \sqrt{m})
$$

if $\alpha^{2}-\beta^{2} m<0$. In both cases $\rho$ equals the product $\rho_{1} \rho_{2}$, where $\rho_{1}$ and $\rho_{2}$ are radii of convergence of (proper) positive rational systems. Consequently, $\rho$ is in $R$, too. 


\section{LIMIT LAWS}

8.1. The classical Drmota-Lalley-Woods theorem. In several applications in combinatorics we are not only interested in a univariate situation, where $z$ is the counting variable but we are interested, too, in a second parameter that we count with the help of another variable (say $u$ ). Hence we are led to consider systems of equations of the form $\mathbf{y}=\mathbf{P}(z, \mathbf{y}, u)$. Of course, if we set $u=1$ we come back to the original counting problem. The next theorem shows that the limiting distribution of the additional parameter is always Gaussian if the system is strongly connected (see $[52])$.

Theorem 8 (Drmota-Lalley-Woods, limiting distribution version: Gaussian limit law for strongly connected systems). Suppose that $\mathbf{y}=\mathbf{P}(z, \mathbf{y}, u)$ is a strongly connected and analytically well defined entire or polynomial system of equations that depends on $u$ and has a solution $\mathbf{f}(z, u)$ that exists in a neighborhood of $u=1$. Furthermore let $h(z, u)$ be given by

$$
h(z, u)=\sum_{n \geq 0} h_{n}(u) z^{n}=H(z, \mathbf{f}(z, u), u),
$$

where $H(z, y, u)$ is entire or a polynomial function with non-negative coefficients that depends on $\mathbf{y}$ and suppose that $h_{n}(u) \neq 0$ for all $n \geq n_{0}$ (for some $n_{0} \geq 0$ ).

Let $X_{n}$ be a random variable which distribution is defined by

$$
\mathbb{E}\left[u^{X_{n}}\right]=\frac{h_{n}(u)}{h_{n}(1)} .
$$

Then $X_{n}$ has a Gaussian limiting distribution. More precisely, we have $\mathbb{E}\left[X_{n}\right]=$ $\mu n+O(1)$ and $\operatorname{Var}\left[X_{n}\right]=\sigma^{2} n+O(1)$ for constants $\mu>0$ and $\sigma^{2} \geq 0$ and

$$
\frac{1}{\sqrt{n}}\left(X_{n}-\mathbb{E}\left[X_{n}\right]\right) \rightarrow N\left(0, \sigma^{2}\right)
$$

8.2. More Gaussian examples, beyond the Drmota-Lalley-Woods case. If the system of equations is not strongly connected then we can still define a random variable $X_{n}$, however, it is not necessarily Gaussian as we will see in the next section. Nevertheless, it is possible to state sufficient conditions, where a Gaussian limiting distribution is present.

Theorem 9 (Gaussian limit law for non-strongly connected systems). Let $\mathbf{y}=$ $\mathbf{P}(z, \mathbf{y}, u)$ be a system of equations as in Theorem 8 with the only difference that it is not strongly connected. Furthermore we assume that the function $h(z, 1)$ is strongly aperiodic.

For every strongly connected component $C_{\ell}$ of the dependency graphs $G$ let $\rho_{\ell}$ denote the radius of convergence of those functions $f_{j}(z, 1)$ that correspond to $C_{\ell}$.

If all $\rho_{\ell}$ are different then $X_{n}$ (defined as in Theorem 8) has a Gaussian limiting distribution.

Proof. We start by setting $u=1$ and check the proof of Theorem 4 . If all the $\rho_{\ell}$ are different then the only cases that can appear are case (2.1) and case (3.1). In all other cases the new radius of convergence is inherited from another function $f_{j}(z)$. Actually the same situation holds if $u$ varies in a sufficiently small neighborhood of 1. By the implicit function theorem it follows that there exists $\rho^{\prime \prime}(u)$ with $\rho\left(f_{j}\left(\rho^{\prime \prime}(u)\right)\right)=\rho^{\prime \prime}(u)$, that is, we get the same singularity structure with a small 
perturbation because of $u$. Actually this is precisely the situation that is needed for the proof of the central limit theorem for $X_{n}$ (see [63]).

8.3. Non-Gaussian limit laws. This section illustrates the wide variety of distributions followed by a parameter in a non-strongly connected grammar.

Theorem 10 (Diversity of possible limit laws for context-free systems). Let $X_{n}$ be the number of occurrences of any given pattern (this pattern could be a given letter!) in a word of length $n$, generated by a grammar (or even by a simpler model of a Markov chain, with an alphabet of 2 letters, each letter having an integer weight). Then $X_{n}$ can follow "any limit law", in the sense that there exist some patterns and some grammars for which the limit curve (for large $n)$ of $\left(k, \operatorname{Prob}\left(X_{n}=k\right)\right)$ can, once rescaled by dividing the length by $n$, be arbitrarily close to any càdlàg curve.

Proof. This is a consequence of the fact that one can get any piecewise-affine function, as proved in [8], so by the Weierstrass theorem one gets any continuous (or càdlàg) distribution. Due to the (possible) periodic behavior of the coefficients of the distribution functions there is also a (possible) periodic behavior of the limiting distribution, that is, for every fixed residue class $\bmod m$ we get different laws. Putting these finitely many limit laws into one figure this leads to a multi-valued curve, as illustrated in Figure 3.

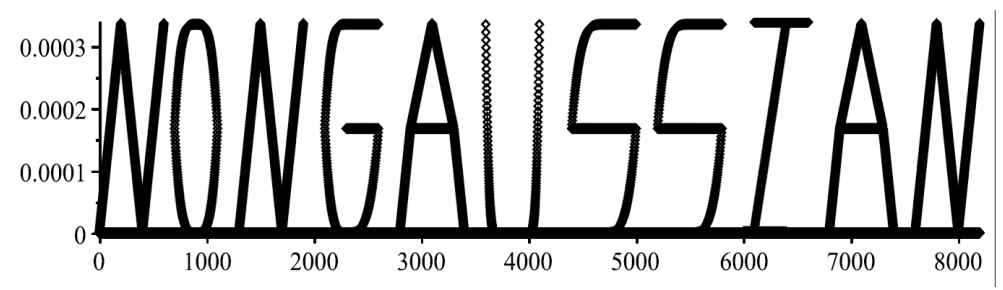

Figure 3. This figure, taken from [8], gives the distribution of the letter "b" in words of length $n=8200$ in a language generated by an ad-hoc regular expression of a few lines. It gives a (discrete) probability distribution which looks like the word "NONGAUSSIAN". The key point of this example is that it is designed such that if one rescales the plot by dividing its width by $n$, then the distribution is converging towards a curve, which still looks like "NONGAUSSIAN". Note that this curve is, at the limit, a curve of a multivalued functional (as can be seen in the O, G, A, S, I letters), however we achieve it for finite length words via a single valued function, by interlacing two sequences mod 2 . This figure illustrates the huge diversity of possible limit laws, even for the distribution of a single letter. It is possible to play the same game starting from continuous distribution instead of discrete distribution. 


\section{Beyond the AlgebraiC CASE: POSITIVE Systems of ENTIRE FUnCTIONS}

Most parts of the analysis of positive polynomial systems of equations also work for positive entire systems; however we have to be careful as the following example shows.

Example 1. The system of equations

$$
\left\{\begin{array}{l}
y_{1}=z\left(e^{y_{2}}+y_{1}\right) \\
y_{2}=z\left(1+2 y_{2} y_{3}\right) \\
y_{3}=z\left(1+y_{3}^{2}\right)
\end{array}\right.
$$

has the following explicit solutions:

$$
\left\{\begin{array}{l}
f_{1}(z)=\frac{z}{1-z} \exp \left(\frac{z}{\sqrt{1-4 z^{2}}}\right) \\
f_{2}(z)=\frac{z}{\sqrt{1-4 z^{2}}} \\
f_{3}(z)=\frac{1-\sqrt{1-4 z^{2}}}{2 z}
\end{array}\right.
$$

Actually we have to be careful with two respects. As we have seen in the proof of Theorem 4 the affine case has to be treated with care. If we just require that our system has no affine subsystem then we can work also in the entire case and obtain just a solution with a singularity of the form (10).

Theorem 11. Let $\mathbf{y}=\mathbf{P}(z, \mathbf{y})$ be an analytically well defined positive system of functional equations, where $\mathbf{P}$ consists of entire functions and we have

$$
\frac{\partial^{2} P_{j}}{\partial y_{j}^{2}} \neq 0, \quad 1 \leq j \leq d .
$$

Then the solutions $f_{j}(z)$ have positive and finite radii of convergence $\rho_{j}$ and a Puiseux critical exponent of the form $2^{-k_{j}}$ with integers $k_{j} \geq 1$, that is, the singular behavior of $f_{j}(z)$ around $\rho_{j}$ is of type

$$
f_{j}(z)=f_{j}\left(\rho_{j}\right)+c_{j}\left(1-z / \rho_{j}\right)^{2^{-k_{j}}}+c_{j}^{\prime}\left(1-z / \rho_{j}\right)^{2 \cdot 2^{-k_{j}}}+\cdots,
$$

where $c_{j} \neq 0$ and where $k_{j}$ is a positive integer.

Instead of assuming condition (20) it is also sufficient that the subsystems $\mathbf{y}_{\ell}=$ $\mathbf{P}_{\ell}\left(z, \mathbf{y}_{\ell}, \mathbf{u}_{\ell}\right)$ (corresponding to the strongly connected component $C_{\ell}$ ) are not affine in $\mathbf{y}_{\ell}$. Indeed, both assumptions are sufficient to obtain a singular expansion of the form $(21)$.

Proof. First of all, Lemma 3 and 4 hold for entire systems of equations. Furthermore the condition (20) ensures that no subsystem is affine. Hence, by checking the proof of Theorem 4, only singularities of type (21) occur. Actually, we only have to go through case (3), and in all these cases we obtain solutions of type (21).

It is possible to cover some cases where affine sub-systems occur. For example, the following theorem ensures that the problem in Example 1 does not occur.

Theorem 12. Let $\mathbf{y}=\mathbf{P}(z, \mathbf{y})$ be an analytically well defined positive system of functional equations, where $\mathbf{P}$ consists of entire functions. Furthermore we assume 
that for each $j=1, \ldots, d$ we either have $\frac{\partial^{2} P_{j}}{\partial y_{j}^{2}} \neq 0$ or if $P_{j}$ is affine in $y_{j}$ then we have

$$
\frac{\partial^{2} P_{j}}{\partial y_{j} \partial y_{i}} \neq 0
$$

for all $i \neq j$ with $\frac{\partial P_{j}}{\partial y_{i}} \neq 0$.

Then the solutions $f_{j}(z)$ have positive and finite radii of convergence $\rho_{j}$. Furthermore, the singular behavior of $f_{j}(z)$ around $\rho_{j}$ and a Puiseux critical exponent of the form $2^{-k_{j}}$ with integers $k_{j} \geq 1$ or of the form $-2^{-k_{j}}$ with integers $k_{j} \geq 0$, that is the singular behavior of $f_{j}(z)$ around $\rho_{j}$ is of type

$$
f_{j}(z)=f_{j}\left(\rho_{j}\right)+c_{j}\left(1-z / \rho_{j}\right)^{2^{-k_{j}}}+c_{j}^{\prime}\left(1-z / \rho_{j}\right)^{2 \cdot 2^{-k_{j}}}+\cdots,
$$

where $c_{j} \neq 0$ and where $k_{j}$ is a positive integer, or of type

$$
f_{j}(z)=\frac{d_{j}}{\left(1-z / \rho_{j}\right)^{2^{-k_{j}}}}+d_{j}^{\prime}+d_{j}^{\prime \prime}\left(1-z / \rho_{j}\right)^{2^{-k_{j}}}+\cdots,
$$

where $d_{j} \neq 0$ and $k_{j}$ are non-negative integers.

Instead of assuming condition (22) it is also sufficient that just the affine subsystems $\mathbf{y}_{\ell}=\mathbf{P}_{\ell}\left(z, \mathbf{y}_{\ell}, \mathbf{u}_{\ell}\right)=\mathbf{A}_{\ell}\left(z, \mathbf{u}_{\ell}\right) \mathbf{y}_{\ell}+\mathbf{B}_{\ell}\left(z, \mathbf{u}_{\ell}\right)$ (corresponding to the strongly connected component $\left.C_{\ell}\right)$ have the property that $\mathbf{A}_{\ell}\left(z, \mathbf{u}_{\ell}\right)$ depends on all components of $\mathbf{u}_{\ell}$.

Proof. By checking the proof of Theorem 4, we observe that we only have to consider the cases (2) and (3). More precisely in case $(2), \delta(z)$ depends on all $y_{j}$ (that correspond to $\left.\mathbf{u}_{j}\right)$.

If we are in case (3), then we obtain a singularity of type (23) as in the proof of Theorem 11.

Finally let us discuss case (2) in more details. For the case (2.1) we obtain a polar singularity $\rho^{\prime \prime}$ that is smaller than all singularities of the functions $f_{j}(z)$. Hence we obtain a singular expansion of type (24) (with $k=0$ ).

For the case (2.2) we obtain a singularity of type (24), where the exponent $2^{-k}$ is inherited from the functions $f_{j}$. Note that the numerator is of type (23) since the denominator depends on all possible functions $f_{j}$ and, thus, no new singularity can appear in the numerator.

Finally for the case (2.3) we obtain a singularity of type (23) that is inherited from the functions $f_{j}(z)$ (of smallest radius of convergence). 


\section{Conclusions}

Now that we have a better picture of the behavior of positive systems of equations and of the asymptotics of the coefficients of the corresponding solutions, several extensions are possible and we plan to say more in future works on the following questions:

- Algorithmic aspects: In order to automate finding the asymptotics, one has to follow the correct branch of the algebraic equations; this is doable by a disjunction of cases following the proof of our main theorem, coupled with an inspection of the associated spectral radii. This leads to a more "algebraic" approach suitable for computer algebra, bypassing some numerical methods like e.g. the Flajolet-Salvy ACA (analytic continuation of algebraic) algorithm [63]. With respect to the Pisot problem (i.e., deciding if one, or an infinite number of $f_{n}$ are zeroes), finding the best equivalent for $\mathbb{N}$-algebraic functions of the Skolem-Mahler-Lech theorem for $\mathbb{N}$-rational functions is also a nice question $[2,19]$. It is also of interest to get algorithms to decide if $f_{n} \geq 0$ for all $n$ [73]. The binomial formula of Section 3 leads to many identities; it is not always easy to predict when the nested sums can be simplified. This has some links with diagonals of rational generating functions.

- Decidability of $\mathbb{N}$-algebraicity: The converse by Soittola $[105,23]$ of a theorem of Berstel [21] shows that it is possible to decide if a rational function is in fact an $\mathbb{N}$-rational function. There is an effective version of this decidability result [16]; Koutschan [79] completed the details and implemented the algorithm. Giving an algorithm to decide in a constructive way if a function is $\mathbb{N}$-algebraic would be nice.

- Extension to differential systems: It is possible to follow a similar approach for linear systems of differential equations, where there is however a broader type of behaviors.

- Extension to infinite systems: If one considers systems having an infinite (countable) number of unknowns $y_{i}(z)$, it is proved in [89] that strongly connected systems also lead to a square-root behavior. It is proved in [54] that the limit law is Gaussian (as soon as a Jacobian operator associated to the system is compact). When the conditions of strong connectivity or of compactness are dropped, many different behaviors may appear, but it is possible to describe interesting subclasses having a regular behavior.

- Extension to attributed grammars: Attribute grammars were introduced by Knuth. Many interesting parameters (like internal path length in trees or area below lattice paths $[12,57,96]$ ) are captured by such grammars. They lead to statistics with a mean which is no longer linear. For a large class of strongly connected positive systems (with a Jacobian condition), it leads to the Airy function, and it is expected that it is also the case for a class of functional equations allowing also negative coefficients.

Acknowledgements The authors acknowledge the financial support of the PHC Amadeus project, the ANR Magnum (ANR-2010-BLAN-0204), the CNRS PEPS Holonomix, and the FWF project SFB Algorithmic and Enumerative Combinatorics (F50). We also thank our two referees for their careful reading of the previous version of this article. 
The genesis of this article is due to Philippe Flajolet. The topic of the paper is in fact very much linked to Philippe Flajolet. The story goes as follows. In the early 90's, Philippe tried to organize at a more international level the communities of people interested in mathematical tools for the average-case analysis of algorithms. This lead to a series of meetings, which soon became the annual international conference "AofA". The first meeting was organized at Dagstuhl in 1993 by Philippe, Helmut Prodinger, and Rainer Kemp; it had many fascinating talks (including e.g. Knuth's presentation of his "giant paper on the giant component", with Janson, Łuczak, and Pittel), and, as it should be with Philippe, many discussions around a glass of beer. During one of these discussions, in the next meeting in 1995, Philippe suggested to Michael Drmota the topic of positive and strongly connected systems of equations, and convinced him to work more also on limit laws. This lead to the article [52], which together with [51] and [55] were the beginning of Drmota's life in analytic combinatorics. Philippe kindly internationally promoted the main theorem from [52] by calling it Drmota-Lalley-Woods theorem, since similar results were obtained independently by Lalley [81] and Woods [118].

In his $\mathrm{PhD}$ thesis [6] under the supervision of Philippe Flajolet, Cyril Banderier considered some enumerative and asymptotics properties of lattice paths and planar maps. The corresponding articles $[9,10,11]$ were the beginning of Banderier's life in analytic combinatorics. Many families of maps and lattice paths have algebraic generating functions. It was then natural to ask if they can be generated by a context-free grammar, and to what kind of asymptotics context-free grammars can lead, beyond the Drmota-Lalley-Woods theorem. Encouraged by Mireille Bousquet-Mélou and Gilles Schaeffer, who also tried to tackle this question [31], he further investigated this problem with his student Hanane Tafat Bouzid, who gave in the chapter 3 of her $\mathrm{PhD}$ thesis [109] a constructive list of critical exponents for $\mathbb{N}$-algebraic functions.

Philippe is also partly responsible for the present general result (dyadic exponents for Puiseux expansion around the radius of convergence). When Michael Drmota presented an incomplete pre-version of this general result in the ANR Boole meeting in May 2010 in Paris, it was again Philippe who asked for an unconditional result for positive polynomial systems. Drmota and Banderier then realized that they were both working on the same problem, so they joined forces for the present article, which is thus naturally dedicated to the memory of Philippe.

\section{REFERENCES}

1. Niels Henrik Abel, Euvres complètes. Tome II, Éditions J. Gabay, Sceaux, 1992, Reprint of the second edition of 1881 .

2. Boris Adamczewski and Jason P. Bell, On vanishing coefficients of algebraic power series over fields of positive characteristic, Invent. Math. 187 (2012), no. 2, 343-393.

3. Michael H. Albert and Michael D. Atkinson, Simple permutations and pattern restricted permutations, Discrete Math. 300 (2005), no. 1-3, 1-15.

4. Jean-Paul Allouche and Jeffrey Shallit, Automatic sequences, Cambridge University Press, Cambridge, 2003, Theory, applications, generalizations.

5. Jean-Michel Autebert, Philippe Flajolet, and Joaquim Gabarró, Prefixes of infinite words and ambiguous context-free languages, Inform. Process. Lett. 25 (1987), no. 4, 211-216.

6. Cyril Banderier, Combinatoire analytique des chemins et des cartes, Phd thesis, Université Paris-VI, jun 2001.

7. Cyril Banderier, Limit laws for basic parameters of lattice paths with unbounded jumps, Mathematics and computer science, II (Versailles, 2002), Trends Math., Birkhäuser, Basel, 2002, pp. 33-47. 
8. Cyril Banderier, Olivier Bodini, Yann Ponty, and Hanane Tafat, On the diversity of pattern distributions in combinatorial systems, Proceedings of Analytic Algorithmics and Combinatorics (ANALCO'12), Kyoto, SIAM, 2012, pp. 107-116.

9. Cyril Banderier, Mireille Bousquet-Mélou, Alain Denise, Philippe Flajolet, Danièle Gardy, and Dominique Gouyou-Beauchamps, Generating functions for generating trees, Discrete Math. 246 (2002), no. 1-3, 29-55, Formal power series and algebraic combinatorics (Barcelona, 1999).

10. Cyril Banderier and Philippe Flajolet, Basic analytic combinatorics of directed lattice paths, Theoret. Comput. Sci. 281 (2002), no. 1-2, 37-80, Selected papers in honour of Maurice Nivat.

11. Cyril Banderier, Philippe Flajolet, Gilles Schaeffer, and Michèle Soria, Random maps, coalescing saddles, singularity analysis, and Airy phenomena, Random Structures Algorithms 19 (2001), no. 3-4, 194-246, Analysis of algorithms (Krynica Morska, 2000).

12. Cyril Banderier and Bernhard Gittenberger, Analytic combinatorics of lattice paths: enumeration and asymptotics for the area, Fourth Colloquium on Mathematics and Computer Science Algorithms, Trees, Combinatorics and Probabilities, Discrete Math. Theor. Comput. Sci. Proc., AG, Assoc. Discrete Math. Theor. Comput. Sci., Nancy, 2006, pp. 345-355.

13. Cyril Banderier and Paweł Hitczenko, Enumeration and asymptotics of restricted compositions having the same number of parts, Discrete Appl. Math. 160 (2012), no. 18, 2542-2554.

14. Cyril Banderier and Donatella Merlini, Lattice paths with an infinite set of jumps, Proceedings of the FPSAC 2002 Conference held in Melbourne, Australia, 2002.

15. Cyril Banderier and Sylviane Schwer, Why Delannoy numbers?, J. Statist. Plann. Inference 135 (2005), no. 1, 40-54.

16. Elena Barcucci, Alberto Del Lungo, Andrea Frosini, and Simone Rinaldi, A technology for reverse-engineering a combinatorial problem from a rational generating function, Adv. in Appl. Math. 26 (2001), no. 2, 129-153.

17. Frédérique Bassino, Mathilde Bouvel, Carine Pivoteau, Adeline Pierrot, and Dominique Rossin, Combinatorial specification of permutation classes, Proceedings of FPSAC'12, Discrete Math. Theor. Comput. Sci. Proc., AR, Assoc. Discrete Math. Theor. Comput. Sci., Nancy, 2012, pp. 791-802.

18. Marie-Pierre Béal, Michel Blocklet, and Cătălin Dima, Zeta functions of finite-type-dyck shifts are $\mathbb{N}$-algebraic, Proceedings of "2014 Information Theory and Applications Workshop" (2014), 8 pp.

19. Jason P. Bell, Stanley N. Burris, and Karen Yeats, On the set of zero coefficients of a function satisfying a linear differential equation, Math. Proc. Cambridge Philos. Soc. 153 (2012), no. 2, 235-247.

20. François Bergeron, Gilbert Labelle, and Pierre Leroux, Combinatorial species and tree-like structures, Encyclopedia of Mathematics and its Applications, vol. 67, Cambridge University Press, Cambridge, 1998, Translated from the 1994 French original by Margaret Readdy, With a foreword by Gian-Carlo Rota.

21. Jean Berstel, Sur les pôles et le quotient de Hadamard de séries $\mathbb{N}$-rationnelles, C. R. Acad. Sci. Paris Sér. A-B 272 (1971), A1079-A1081.

22. Jean Berstel and Luc Boasson, Towards an algebraic theory of context-free languages, Fund. Inform. 25 (1996), no. 3-4, 217-239.

23. Jean Berstel and Christophe Reutenauer, Noncommutative rational series with applications, Encyclopedia of Mathematics and its Applications, vol. 137, Cambridge University Press, Cambridge, 2011.

24. Alberto Bertoni, Massimiliano Goldwurm, and Massimo Santini, Random generation for finitely ambiguous context-free languages, Theor. Inform. Appl. 35 (2001), no. 6, 499-512 (2002), A tribute to Aldo de Luca.

25. Frits Beukers and Gert Heckman, Monodromy for the hypergeometric function ${ }_{n} F_{n-1}$, Invent. Math. 95 (1989), no. 2, 325-354.

26. Olivier Bodini, Alexis Darrasse, and Michèle Soria, Distances in random Apollonian network structures, 20th Annual International Conference on Formal Power Series and Algebraic Combinatorics (FPSAC 2008), Discrete Math. Theor. Comput. Sci. Proc., AJ, Assoc. Discrete Math. Theor. Comput. Sci., Nancy, 2008, pp. 307-318. 
27. Olivier Bodini and Yann Ponty, Multi-dimensional boltzmann sampling of languages, 21st International Meeting on Probabilistic, Combinatorial, and Asymptotic Methods in the Analysis of Algorithms (AofA'10), Discrete Math. Theor. Comput. Sci. Proc., AM, Assoc. Discrete Math. Theor. Comput. Sci., Nancy, 2010, pp. 49-64.

28. Alin Bostan, Frédéric Chyzak, Grégoire Lecerf, Bruno Salvy, and Éric Schost, Differential equations for algebraic functions, Proceedings of ISSAC 2007, ACM, 2007.

29. Alin Bostan and Manuel Kauers, Automatic Classification of Restricted Lattice Walks, Proceedings of FPSAC'09 (Christian Krattenthaler, Volker Strehl, and Manuel Kauers, eds.), 2009, pp. 201-215.

30. Alin Bostan and Manuel Kauers, The complete generating function for Gessel walks is algebraic, Proc. Amer. Math. Soc. 138 (2010), no. 9, 3063-3078, With an appendix by Mark van Hoeij.

31. Mireille Bousquet-Mélou, Rational and algebraic series in combinatorial enumeration, International Congress of Mathematicians. Vol. III, Eur. Math. Soc., Zürich, 2006, pp. 789-826.

32. Mireille Bousquet-Mélou and Arnaud Jehanne, Polynomial equations with one catalytic variable, algebraic series and map enumeration, J. Combin. Theory Ser. B 96 (2006), no. 5, 623-672.

33. Mireille Bousquet-Mélou and Gilles Schaeffer, Walks on the slit plane, Probab. Theory Related Fields 124 (2002), no. 3, 305-344.

34. Benjamin Canou and Alexis Darrasse, Fast and sound random generation for automated testing and benchmarking in objective Caml, Proceedings of the 2009 ACM SIGPLAN workshop on ML (New York, NY, USA), ML'09, 2009, pp. 61-70.

35. Tullio Ceccherini-Silberstein and Wolfgang Woess, Growth and ergodicity of context-free languages, Trans. Amer. Math. Soc. 354 (2002), no. 11, 4597-4625.

36. _ Growth-sensitivity of context-free languages, Theoret. Comput. Sci. 307 (2003), no. 1, 103-116, Words.

37. _ Context-free pairs of groups I: Context-free pairs and graphs, European J. Combin. 33 (2012), no. 7, 1449-1466.

38. Noam Chomsky and Marcel-Paul Schützenberger, The algebraic theory of context-free languages, Computer programming and formal systems, North-Holland, Amsterdam, 1963, pp. 118-161.

39. Gilles Christol, Teturo Kamae, Michel Mendès France, and Gérard Rauzy, Suites algébriques, automates et substitutions, Bull. Soc. Math. France 108 (1980), no. 4, 401-419.

40. David Volfovich Chudnovsky and Gregory Volfovich Chudnovsky, On expansion of algebraic functions in power and Puiseux series. II, J. Complexity 3 (1987), no. 1, 1-25.

41. Peter Clote, Yann Ponty, and Jean-Marc Steyaert, Expected distance between terminal nucleotides of RNA secondary structures, J. Math. Biol. 65 (2012), no. 3, 581-599.

42. James Cockle, On transcendental and algebraic solution, Philosophical Magazine XXI (1861), 379-383.

43. Louis Comtet, Calcul pratique des coefficients de Taylor d'une fonction algébrique, Enseignement Math. (2) 10 (1964), 267-270.

44. _ Advanced combinatorics, D. Reidel Publishing Co., Dordrecht, 1974, enlarged edition of the 2 volumes "Analyse combinatoire" published in French in 1970, by Presses Universitaires de France.

45. Alexis Darrasse, Random XML sampling the Boltzmann way, http://arxiv.org/pdf/0807.0992v1.pdf (2008), 6 pp.

46. M. Delest, Algebraic languages: a bridge between combinatorics and computer science, Formal power series and algebraic combinatorics (New Brunswick, NJ, 1994), DIMACS Ser. Discrete Math. Theoret. Comput. Sci., vol. 24, Amer. Math. Soc., Providence, RI, 1996, pp. $71-87$.

47. Marie-Pierre Delest and Gérard Viennot, Algebraic languages and polyominoes enumeration, Theoret. Comput. Sci. 34 (1984), no. 1-2, 169-206.

48. Jan Denef and Leonard Lipshitz, Power series solutions of algebraic differential equations, Math. Ann. 267 (1984), no. 2, 213-238.

49. Alain Denise, Yann Ponty, and Michel Termier, Random generation of structured genomic sequences, RECOMB'2003, Berlin, April 2003, 2003, p. 3 pages (poster).

50. Alain Denise and Paul Zimmermann, Uniform random generation of decomposable structures using floating-point arithmetic, Theoret. Comput. Sci. 218 (1999), no. 2, 233-248, Caen '97. 
51. Michael Drmota, Asymptotic distributions and a multivariate darboux method in enumeration problems, J. Combinatorial Theory, Ser. A 67 (1994), 169-184.

52. _ Systems of functional equations, Random Structures Algorithms 10 (1997), no. 1-2, 103-124, Average-case analysis of algorithms (Dagstuhl, 1995).

53. _ Random trees, Springer, Vienna, 2009, An interplay between combinatorics and probability.

54. Michael Drmota, Bernhard Gittenberger, and Johannes F. Morgenbesser, Infinite systems of functional equations and Gaussian limiting distributions, 23rd International Meeting on Probabilistic, Combinatorial, and Asymptotic Methods in the Analysis of Algorithms (AofA'12), Discrete Math. Theor. Comput. Sci. Proc., AQ, Assoc. Discrete Math. Theor. Comput. Sci., Nancy, 2012, pp. 453-478.

55. Michael Drmota and Michèle Soria, Marking in combinatorial constructions: generating functions and limiting distributions, Theor. Comput. Sci. 144 (1995), 67-99.

56. Manfred Droste, Werner Kuich, and Heiko Vogler (eds.), Handbook of weighted automata, Monographs in Theoretical Computer Science. An EATCS Series, Springer, Berlin, 2009.

57. Philippe Duchon, q-grammars and wall polyominoes, Ann. Comb. 3 (1999), no. 2-4, 311-321, On combinatorics and statistical mechanics.

58. _ On the enumeration and generation of generalized Dyck words, Discrete Math. $\mathbf{2 2 5}$ (2000), no. 1-3, 121-135, Formal power series and algebraic combinatorics (Toronto, ON, 1998).

59. Philippe Duchon, Philippe Flajolet, Guy Louchard, and Gilles Schaeffer, Boltzmann samplers for the random generation of combinatorial structures, Combin. Probab. Comput. 13 (2004), no. 4-5, 577-625.

60. Philippe Flajolet, Analytic models and ambiguity of context-free languages, Theoret. Comput. Sci. 49 (1987), no. 2-3, 283-309, Twelfth international colloquium on automata, languages and programming (Nafplion, 1985).

61. Philippe Flajolet and Marc Noy, Analytic combinatorics of non-crossing configurations, Discrete Math. 204 (1999), no. 1-3, 203-229.

62. Philippe Flajolet and Andrew Odlyzko, Singularity analysis of generating functions, SIAM J. Discrete Math. 3 (1990), no. 2, 216-240.

63. Philippe Flajolet and Robert Sedgewick, Analytic combinatorics, Cambridge University Press, Cambridge, 2009.

64. Philippe Flajolet, Paul Zimmerman, and Bernard Van Cutsem, A calculus for the random generation of labelled combinatorial structures, Theoret. Comput. Sci. 132 (1994), no. 1-2, $1-35$.

65. Harry Furstenberg, Algebraic functions over finite fields, J. Algebra 7 (1967), 271-277.

66. Ian P. Goulden and David M. Jackson, Combinatorial enumeration, Dover Publications Inc., Mineola, NY, 2004, With a foreword by Gian-Carlo Rota, Reprint of the 1983 original.

67. Rostislav Grigorchuk and Pierre de la Harpe, On problems related to growth, entropy, and spectrum in group theory, J. Dynam. Control Systems 3 (1997), no. 1, 51-89.

68. Robert Harley, On the theory of the transcendental solution of algebraic equations, Quart. Journal of Pure and Applied Math 5 (1862), 337-361.

69. William A. Harris, Jr. and Yasutaka Sibuya, The reciprocals of solutions of linear ordinary differential equations, Adv. in Math. 58 (1985), no. 2, 119-132.

70. Timothy Hickey and Jacques Cohen, Uniform random generation of strings in a context-free language, SIAM J. Comput. 12 (1983), no. 4, 645-655.

71. André Joyal, Une théorie combinatoire des séries formelles, Adv. in Math. 42 (1981), no. 1, $1-82$.

72. Reinwald Jungen, Sur les séries de Taylor n'ayant que des singularités algébricologarithmiques sur leur cercle de convergence, Comment. Math. Helv. 3 (1931), no. 1, 266306.

73. Manuel Kauers and Veronika Pillwein, When can we decide that a p-finite sequence is positive?, Proceedings of ISSAC'10 (2010), 195-202, arXiv 1005.0600.

74. R. Kemp, A note on the density of inherently ambiguous context-free languages, Acta Inform. 14 (1980), no. 3, 295-298.

75. Rainer Kemp, Random multidimensional binary trees, Journal of Information Processing and Cybernetics (EIK) $\mathbf{2 9}$ (1993), 9-36 
76. Stephen Cole Kleene, Representation of events in nerve nets and finite automata, Automata studies, Annals of mathematics studies, no. 34, Princeton University Press, Princeton, N. J., 1956, pp. 3-41.

77. Donald E. Knuth, The art of computer programming. Vol. 2: Seminumerical algorithms. 3rd ed., Addison-Wesley. xiii+762 p., 1998, (2: 1981, 1:1969).

78. Maxim Kontsevich, Noncommutative identities, http://arxiv.org/abs/1109.2469v1 (2011), $10 \mathrm{pp}$.

79. Christoph Koutschan, Regular languages and their generating functions: the inverse problem, Theoret. Comput. Sci. 391 (2008), no. 1-2, 65-74.

80. Joseph-Louis Lagrange, Nouvelle méthode pour résoudre les équations littérales par le moyen des séries, Histoire de l'Académie Royale des Sciences et des Belles Lettres de Berlin avec les Mémoires tirez des registres de cette Académie XXIV (1770), 251-326, (Reprinted in Joseph Louis de Lagrange - Euvres complètes, tome 3. Paris: Gauthier-Villars, pp. 5-73, 1869.).

81. Steven P. Lalley, Finite range random walk on free groups and homogeneous trees, The Annals of Probability 21 (1993), no. 4, 571-599.

82. Steven P. Lalley, Algebraic systems of generating functions and return probabilities for random walks, Dynamics and randomness II, Nonlinear Phenom. Complex Systems, vol. 10, Kluwer Acad. Publ., Dordrecht, 2004, pp. 81-122.

83. Wolfdieter Lang, On generalizations of the Stirling number triangles, J. Integer Seq. 3 (2000), no. 2, Article 00.2.4.

84. M. Lothaire, Applied combinatorics on words, Encyclopedia of Mathematics and its Applications, vol. 105, Cambridge University Press, Cambridge, 2005, A collective work by Jean Berstel, Dominique Perrin, Maxime Crochemore, Eric Laporte, Mehryar Mohri, Nadia Pisanti, Marie-France Sagot, Gesine Reinert, Sophie Schbath, Michael Waterman, Philippe Jacquet, Wojciech Szpankowski, Dominique Poulalhon, Gilles Schaeffer, Roman Kolpakov, Gregory Koucherov, Jean-Paul Allouche and Valérie Berthé, With a preface by Berstel and Perrin.

85. Toufik Mansour and Mark Shattuck, Pattern avoiding partitions, sequence A054391 and the kernel method, Appl. Appl. Math. 6 (2011), no. 12, 397-411.

86. Fredrik Johansson Manuel Kauers, Maximilian Jaroschek, Ore polynomials in Sage, Computer Algebra and Polynomials, Lecture Notes in Computer Science (2014), 16 pp., arXiv 1306.4263.

87. A. Meir and J. W. Moon, On the altitude of nodes in random trees, Canad. J. Math. 30 (1978), no. 5, 997-1015.

88. Basile Morcrette, Fully analyzing an algebraic pólya urn model, Proceedings of LATIN 2012, LNCS 7256, Springer, 2012, pp. 568-581.

89. Johannes F. Morgenbesser, Square root singularities of infinite systems of functional equations, 21st International Meeting on Probabilistic, Combinatorial, and Asymptotic Methods in the Analysis of Algorithms (AofA'10), Discrete Math. Theor. Comput. Sci. Proc., AM, Assoc. Discrete Math. Theor. Comput. Sci., Nancy, 2010, pp. 513-525.

90. David E. Muller and Paul E. Schupp, Context-free languages, groups, the theory of ends, second-order logic, tiling problems, cellular automata, and vector addition systems, Bull. Amer. Math. Soc. (N.S.) 4 (1981), no. 3, 331-334.

91. Alois Panholzer, Gröbner bases and the defining polynomial of a context-free grammar generating function, J. Autom. Lang. Comb. 10 (2005), no. 1, 79-97.

92. Ion Petre and Arto Salomaa, Chapter 7: Algebraic systems and pushdown automata, Handbook of weighted automata, Monogr. Theoret. Comput. Sci. EATCS Ser., Springer, Berlin, 2009, pp. 257-289.

93. Pavel A. Pevzner and Michael S. Waterman, Open combinatorial problems in computational molecular biology, Third Israel Symposium on the Theory of Computing and Systems (Tel Aviv, 1995), IEEE Comput. Soc. Press, Los Alamitos, CA, 1995, pp. 158-173.

94. Carine Pivoteau, Bruno Salvy, and Michèle Soria, Algorithms for combinatorial structures: Well-founded systems and Newton iterations, J. Combin. Theory Ser. A 119 (2012), no. 8, 1711-1773.

95. Christophe Reutenauer and Marco Robado, On an algebraicity theorem of kontsevich, DMTCS Proceedings 0 (2012), no. 01, 239-246, Conference FPSAC 2012, Nagoya. 
96. Christoph Richard, Limit distributions and scaling functions, Polygons, polyominoes and polycubes, Lecture Notes in Phys., vol. 775, Springer, Dordrecht, 2009, pp. 247-299.

97. Grzegorz Rozenberg and Arto Salomaa (eds.), Handbook of formal languages, Springer, Berlin, 1997, 3 volumes.

98. Arto Salomaa, Formal languages and power series, Handbook of theoretical computer science, Vol. B, Elsevier, Amsterdam, 1990, pp. 103-132.

99. Arto Salomaa and Matti Soittola, Automata-theoretic aspects of formal power series, Springer, New York, 1978, Texts and Monographs in Computer Science.

100. Carsten Schneider, A Symbolic Summation Approach to Find Optimal Nested Sum Representations, Motives, Quantum Field Theory, and Pseudodifferential Operators (A. Carey, D. Ellwood, S. Paycha, and S. Rosenberg, eds.), Clay Mathematics Proceedings, vol. 12, Amer. Math. Soc, 2010, arXiv:0904.2323, pp. 285-308 (english).

101. Marcel-Paul Schützenberger, On a theorem of R. Jungen, Proc. Amer. Math. Soc. 13 (1962), 885-890.

102. Hermann Amandus Schwarz, On these cases in which the Gaussian hypergeometric series represents an algebraic function of its fourth element. (Ueber diejenigen Fälle, in welchen die Gauss'sche hypergeometrische Reihe eine algebraische Function ihres vierten Elementes darstellt.), Journal für die Reine und Angewandte Mathematik LXXV (1872), 292-335.

103. Michael F. Singer, Algebraic solutions of nth order linear differential equations, Proceedings of the Queen's Number Theory Conference, 1979 (Kingston, Ont., 1979) (Kingston, Ont.), Queen's Papers in Pure and Appl. Math., vol. 54, Queen's Univ., 1980, pp. 379-420.

104. __ Algebraic relations among solutions of linear differential equations, Trans. Amer. Math. Soc. 295 (1986), no. 2, 753-763.

105. Matti Soittola, Positive rational sequences, Theoret. Comput. Sci. 2 (1976), no. 3, 317-322.

106. Alan D. Sokal, A ridiculously simple and explicit implicit function theorem, Sém. Lothar. Combin. 61A (2009/10), Art. B61Ad, 21.

107. Richard P. Stanley, Enumerative combinatorics. Vol. 2, Cambridge Studies in Advanced Mathematics, vol. 62, Cambridge University Press, Cambridge, 1999, With a foreword by Gian-Carlo Rota and appendix 1 by Sergey Fomin.

108. Bernd Sturmfels, Solving systems of polynomial equations, CBMS Regional Conference Series in Mathematics, vol. 97, Published for the Conference Board of the Mathematical Sciences, Washington, DC, 2002.

109. Hanane Tafat Bouzid, Combinatoire analytique des langages réguliers et algébriques, Phd thesis, Université Paris-XIII, Dec 2012.

110. Jules Tannery, Propriétés des intégrales des équations différentielles linéaires à coefficients variables, Thèse de doctorat ès sciences mathématiques, Faculté des Sciences de Paris, 1874, Available at http://gallica.bnf.fr.

111. François Trèves, Topological vector spaces, distributions and kernels, Dover Publications Inc., Mineola, NY, 2006, Unabridged republication of the 1967 original.

112. A. J. van der Poorten, Some facts that should be better known, especially about rational functions, Number theory and applications (Banff, AB, 1988), NATO Adv. Sci. Inst. Ser. C Math. Phys. Sci., vol. 265, Kluwer Acad. Publ., Dordrecht, 1989, pp. 497-528. MR 1123092 (92k:11011)

113. Jan van Leeuwen (ed.), Handbook of theoretical computer science. Vol. B, Elsevier Science Publishers B.V., Amsterdam, 1990, Formal models and semantics.

114. Gérard Viennot, Enumerative combinatorics and algebraic languages, Fundamentals of computation theory (Cottbus, 1985), Lecture Notes in Comput. Sci., vol. 199, Springer, Berlin, 1985, pp. 450-464.

115. Michael S. Waterman, Applications of combinatorics to molecular biology, Handbook of combinatorics, Vol. 1, 2, Elsevier, Amsterdam, 1995, pp. 1983-2001.

116. Frank Weinberg and Markus E. Nebel, Extending stochastic context-free grammars for an application in bioinformatics, Language and automata theory and applications, Lecture Notes in Comput. Sci., vol. 6031, Springer, Berlin, 2010, pp. 585-595.

117. Wolfgang Woess, Context-free pairs of groups II-cuts, tree sets, and random walks, Discrete Math. 312 (2012), no. 1, 157-173.

118. Alan R. Woods, Coloring rules for finite trees, and probabilities of monadic second order sentences, Random Struct. Algorithms 10 (1997), no. 4, 453-485. 